\title{
On reducing the splitting error in Yosida methods for the Navier-Stokes equations with grad-div stabilization
}

\author{
Leo G. Rebholz* $\quad$ Mengying Xiao ${ }^{\dagger}$
}

\begin{abstract}
This paper analyzes the accuracy of the 'discretize-then-split' Yosida solver for incompressible flow problems, when divergence-free elements are used together with grad-div stabilization (with parameter $\gamma$ ). The Yosida method uses an inexact block LU factorization to create linear algebraic systems that are easier to solve, but at the expense of accuracy. We prove the difference between solutions of the exact and approximated linear algebraic systems is $O\left(\gamma^{-2}\right)$ in the natural norms of the associated finite element problems, and thus that full accuracy can be obtained by the Yosida method if large $\gamma$ is used $(\gamma \geq 10$ is sufficient in our numerical examples). The proof is based on transforming the Yosida inexact linear algebraic system into finite element problems, and analyzing these problems with finite element techniques based on pointwise divergence-free subspaces and their orthogonal complements.
\end{abstract}

\section{Introduction}

It is well known that solving coupled velocity-pressure saddle point linear algebraic systems that arise from finite element spatial discretizations for Navier-Stokes (NS) and related equations can be very challenging. Consider the following problem of finding a velocity $u$ and pressure $p$ in a domain $\Omega \subset \mathbb{R}^{d}(\mathrm{~d}=2,3)$ satisfying

$$
\begin{aligned}
\sigma u+U \cdot \nabla u+\nabla p-\nu \Delta u & =f \\
\nabla \cdot u & =0, \\
\left.u\right|_{\partial \Omega} & =g,
\end{aligned}
$$

where $\nu$ is the viscosity, $\sigma$ typically represents a constant that is inversely proportional to a timestep size, $g$ is a Dirichlet boundary condition, $f$ is a forcing and possibly combined with known velocity terms, and $U$ is a given velocity field (often a previous iteration in a steady NS scheme, or is derived from previous timesteps in a time dependent NS scheme).

After applying a finite element discretization, a linear system that arises taking the form

$$
\left(\begin{array}{cc}
A & B \\
B^{T} & 0
\end{array}\right)\left(\begin{array}{c}
U \\
P
\end{array}\right)=\left(\begin{array}{c}
F \\
G
\end{array}\right)
$$

\footnotetext{
*Department of Mathematical Sciences, Clemson University, Clemson, SC 29634 (rebholz@clemson.edu), partially supported by NSF grant DMS1112593 and U.S. Army grant 65294-MA.

${ }_{\dagger}^{\dagger}$ Department of Mathematical Sciences, Clemson University, Clemson, SC 29634 (mengyix@clemson.edu), partially supported by NSF grant DMS1112593.
} 
must be solved. Performing a block LU decomposition, we observe the problem can be written equivalently as

$$
\left(\begin{array}{cc}
A & 0 \\
B^{T} & -B^{T} A^{-1} B
\end{array}\right)\left(\begin{array}{cc}
I & A^{-1} B \\
0 & I
\end{array}\right)\left(\begin{array}{c}
U \\
P
\end{array}\right)=\left(\begin{array}{c}
F \\
G
\end{array}\right)
$$

revealing that a fundamental problem in solving this system is the Schur complement matrix $S:=$ $B^{T} A^{-1} B$. If the $A$ matrix is close to symmetric positive definite, as it would be if $\nu$ or $\sigma$ is large, then the stiffness (or mass) matrix would dominate the (nonsymmetric) convective contributions to $A$ arising from $U \cdot \nabla u$, and solving with $S$ becomes less difficult. However, when the convective contributions make up a significant part of $A$, then the linear algebraic problem can be very difficult. Although significant progress has been made in recent years for solving such linear systems, e.g. $[3,8,2]$, for many problems with small $\nu$ or anisotropic meshes, it remains very difficult to find robust and efficient solvers, without taking excessively small timestep sizes.

A popular approach to circumvent this difficulty is the Yosida 'discretize-then-split' approach $[26,27,23]$ (and its variants, see e.g. [28]). These methods split the linear algebraic system with an inexact block LU factorization in order to create a symmetric positive definite (SPD) Schur complement, but while preserving the momentum equation. For the classical first order Yosida method, if we consider the $A$ block to be comprised of $A=\widetilde{A}+N$, where $\widetilde{A}$ is SPD and $N$ includes the contributions from the convective term, the inexact Yosida factorization is done via

$$
\left(\begin{array}{cc}
A & B \\
B^{T} & 0
\end{array}\right) \approx\left(\begin{array}{cc}
A & 0 \\
B^{T} & -B^{T} \widetilde{A}^{-1} B
\end{array}\right)\left(\begin{array}{cc}
I & A^{-1} B \\
0 & I
\end{array}\right)=\left(\begin{array}{cc}
A & B \\
B^{T} & B^{T}\left(A^{-1}-\widetilde{A}^{-1}\right) B
\end{array}\right) .
$$

Here, the (approximated) Schur complement $\widetilde{S}:=B^{T} \widetilde{A}^{-1} B$ is SPD and is generally much easier to solve with than $S$. An interesting and important feature of the Yosida splitting is that it does not alter the algebraic momentum equation, only the algebraic continuity equation, which can be seen from the fact that the first row of the approximated block system is that same as the original. Clearly, the error in the inexact factorization comes from the approximation $A^{-1} \approx \widetilde{A}^{-1}$, which is shown to be $O\left(\sigma^{-2}\right)$ in [28] for the time-dependent NS equations. We note even higher order accuracy is possible if additional linear solves are performed, via the pressure-correction Yosida method [28].

The purpose of this paper is to show that in the setting of divergence-free mixed finite elements used together with grad-div stabilization with penalty parameter $\gamma$ (noting that the stabilization is not negligible due to the inexact factorization), Yosida methods can obtain highly accurate solutions, even for smaller $\gamma$ or for small (or 0) $\sigma$. We prove solutions to this 'Yosida-penalty method' converge to the solution of the original linear algebraic system with rate $O\left(\gamma^{-2}\right)$ as $\gamma \rightarrow$ $\infty$ (independent of the mesh width $h$ ), in the natural norms of the corresponding finite element problem. The general idea of the method is that the error in the splitting is restricted to the continuity equation, and using grad-div stabilization in the momentum equation will help to regain mass conservation through penalization. The idea of the proof is to transfer the systems that arise from the inexact Yosida factorization into finite element problems, and perform the analysis in that framework.

This paper is arranged as follows: Section 2 presents notation and mathematical preliminaries to make for a smoother analysis in later sections. Section 3 presents the Yosida-penalty method, convergence analysis for it, as well as several numerical tests. In section 4 , we explore the connection between the Yosida-penalty method and the classical iterated penalty method, and compare the methods on some benchmark tests. 


\subsection{Connection to projection methods}

Yosida 'discretize-then-split' splitting methods are one approach to avoid having to solve the exact linear algebraic systems in NS and related equations, but there are others. Probably the most popular method is the 'split-then-discretize' approach pioneered by Chorin and Temam [6, 30] in the 1960's for time-dependent NS problems, and improved on in numerous ways since then [13, 25, 24, 4, 16]. These methods are called projection methods and are based on a Hodge decomposition that splits the system in the temporal discretization (before the spatial discretization is applied).

A similar result to that proven herein for the Yosida method is established for projection methods in [20]: If a projection method is enhanced with grad-div stabilization (called penalty-projection method [16] ) on a suitable mesh, then the coupled method (i.e. unsplit) solution is recovered as $\gamma \rightarrow \infty$, with convergence rate $O\left(\gamma^{-1}\right)$. In other words, this paper completes a circle of ideas that grad-div stabilization, when used in an appropriate discrete setting, effectively removes splitting error both in 'discretize-then-split' and 'split-then-discretize' methods.

An interesting difference in the Yosida-penalty result herein is that the convergence to the coupled method solution is $O\left(\gamma^{-2}\right)$. The essential reason is that Yosida-penalty does two solves with the $A$ matrix and one solve with an SPD Schur complement, while the penalty-projection method does just one solve with an $A$ matrix and one SPD Schur complement solve. Thus, the grad-div stabilization (penalty) is applied twice in a Yosida solve, and only once in a projection solve. However, the penalty-projection method could be altered to do two steps of a penaltyiteration, which would i) make it the same cost as Yosida-penalty and ii) make the convergence $O\left(\gamma^{-2}\right)$. This is discussed in more detail in section 4 .

\section{Mathematical preliminaries}

We consider a domain $\Omega \subset \mathbb{R}^{d}(d=2,3)$ that is open, connected, and with Lipschitz boundary $\partial \Omega$. The $L^{2}(\Omega)$ norm and inner product will be denoted by $\|\cdot\|$ and $(\cdot, \cdot)$, and $L_{0}^{2}(\Omega)$ denotes the zero mean subspace of $L^{2}(\Omega)$. Throughout this paper, it is understood by context whether a particular space is scalar or vector valued, and so we do not distinguish notation.

For the natural velocity and pressure spaces for Stokes and NSE, we will use the notation

$$
X:=H_{0}^{1}(\Omega), \quad Q:=L_{0}^{2}(\Omega) .
$$

In the space $X$, the Poincare inequality is known to hold: There exists $\lambda>0$, dependent only on the size of $\Omega$, such that for every $v \in X$,

$$
\|v\| \leq \lambda\|\nabla v\| .
$$

The dual space of $X$ will be denoted by $X^{\prime}$, with norm $\|\cdot\|_{-1}$.

Let $\tau_{h}$ be a conforming, shape-regular, and simplicial triangulation of $\Omega$ with $h_{T}$ denoting the maximum element diameter. We denote with $P_{k}$ the space of degree $k$ globally continuous piecewise polynomials with respect to $\tau_{h}$, and $P_{k}^{d i s c}$ the space of degree $k$ piecewise polynomials that can be discontinuous across elements.

Throughout the paper, we consider only discrete velocity-pressure spaces $\left(X_{h}, Q_{h}\right) \subset(X, Q)$ that satisfy the LBB condition: there exists a constant $\beta$, independent of $h$, satisfying

$$
\inf _{q \in Q_{h}} \sup _{v \in X_{h}} \frac{(\nabla \cdot v, q)}{\|q\|\|\nabla v\|} \geq \beta>0 .
$$


We also assume the spaces satisfy the inclusion $\nabla \cdot X_{h} \subset Q_{h}$. A common examples of such elements are the $\left(P_{k}, P_{k-1}^{\text {disc }}\right)$ Scott-Vogelius $(\mathrm{SV})$ elements on meshes with particular structure [33, 1], and see $[9,15]$ for more examples.

Define the space of discrete divergence-free functions by

$$
V_{h}:=\left\{v \in X_{h}:(\nabla \cdot v, q)=0 \forall q \in Q_{h}\right\}
$$

From our assumptions, functions in $V_{h}$ are pointwise divergence-free; that is,

$$
V_{h}=\left\{v \in X_{h}:\|\nabla \cdot v\|=0\right\} .
$$

Element choices $\left(X_{h}, Q_{h}\right)$ whose discretely divergence-free subspaces are also pointwise divergence are typically referred to as divergence-free elements.

Define $R_{h}:=V_{h}^{\perp} \subset X_{h}$ to be the orthogonal complement of $V_{h}$ with respect to the $H_{0}^{1}$ inner product, i.e., $v \in R_{h}$ if $(\nabla v, \nabla w)=0$ for all $w \in V_{h}$. The following lemma concerning the norm on $R_{h}$ is proven in a general case in [12], and a shorter proof for our particular choice of divergence-free elements is given in [20]. It shows the divergence norm on $R_{h}$ is equivalent to the gradient norm, with the constant being dependent on the inverse of the inf-sup constant.

Lemma 2.1. Let $\left(X_{h}, Q_{h}\right) \subset(X, Q)$ satisfy the inf-sup condition and $\nabla \cdot X_{h} \subset Q_{h}$. Then for every $r_{h} \in R_{h}$,

$$
\left\|\nabla r_{h}\right\| \leq \beta^{-1}\left\|\nabla \cdot r_{h}\right\|
$$

Define the skew-symmetric, trilinear operator $b^{*}: X \times X \times X \rightarrow \mathbb{R}$ by

$$
b^{*}(u, v, w):=\frac{1}{2}(u \cdot \nabla v, w)-\frac{1}{2}(u \cdot \nabla w, v),
$$

and recall, from e.g. [12], that there exists $C_{s}$ depending only on $\Omega$ such that

$$
\left|b^{*}(u, v, w)\right| \leq C_{s}\|\nabla u\|\|\nabla v\|\|\nabla \nabla\|,
$$

for every $u, v, w \in X$.

Define the bilinear form $a: X \times X \rightarrow \mathbb{R}$ by:

$$
a(u, v):=\sigma(u, v)+\nu(\nabla u, \nabla v)+b^{*}(U, u, v),
$$

where $U \in X_{h}$ is known and we assume $\|\nabla U\| \leq C_{U}$, and $\sigma$ is inversely proportional to the timestep size. For a steady NS problem, $\sigma=0$ and $U=u_{h}^{\text {prev }}$ from a previous iteration is a typical choice, and for an unsteady problem using linearized BDF2 timestepping, $U=2 u_{h}^{n-1}-u_{h}^{n-2}$ and $\sigma=\frac{3}{2 \Delta t}$ is a common choice.

The bilinear form $a$ is easily seen to be continuous and coercive, and we denote the $a$-norm by

$$
\|u\|_{a}:=a(u, u)^{1 / 2}=\sigma\|u\|^{2}+\nu\|\nabla u\|^{2} .
$$

The $a$-norm is equivalent to the $H_{0}^{1}$ norm, independent of $h$, as is verified easily since

$$
\nu\|\nabla u\|^{2} \leq\|u\|_{a}^{2} \leq\left(\nu+\sigma \lambda^{2}\right)\|\nabla u\|^{2} .
$$


We will also analyze the methods herein using the bilinear form $\widetilde{a}: X \times X \rightarrow \mathbb{R}$ by:

$$
\widetilde{a}(u, v):=\sigma(u, v)+\nu(\nabla u, \nabla v) .
$$

This bilinear form is symmetric, continuous and coercive, and defines an inner product on $X$. We note that $a(u, u)=\widetilde{a}(u, u)$, and we use the same notation for $\|u\|_{a}=\|u\|_{\tilde{a}}=(\widetilde{a}(u, u))^{1 / 2}$.

Our analysis uses an analogous result to Lemma 2.1 , but based on the $\tilde{a}$-inner product and norm. For this purpose, define the space orthogonal to $V_{h}$ in the $a$-inner product to be

$$
R_{h}^{\tilde{a}}=\left\{w \in X_{h}: \widetilde{a}(w, v)=0, \forall v \in V_{h}\right\}
$$

so that $X_{h}=V_{h} \oplus R_{h}^{\tilde{a}}$ in the sense of the $\tilde{a}$-inner product.

Lemma 2.2. For every $r \in R_{h}^{\tilde{a}}$, there exists $C_{\tilde{a}}:=\beta^{-1}\left(\sigma \lambda^{2}+\nu\right)$ such that

$$
\|r\|_{\tilde{a}} \leq C_{\tilde{a}}\|\nabla \cdot r\|
$$

Proof. This follows from the inf-sup condition on $\left(X_{h}, Q_{h}\right)$ with the bilinear form $\tilde{a}$, thanks to (2.3).

\section{The Yosida-penalty method}

We now present, analyze and test the Yosida-penalty method. For simplicity, we restrict to the most basic case, i.e. without the improvements such as pressure-correction methods which are discussed in $[27,26,11,28,5]$. Extension of the ideas of this paper can be made to those techniques with similar results (in terms of behavior with respect to $\gamma$ ), but we note that no further improvements arise in the other methods; i.e. the convergence rate with respect to $\gamma$ is the same. Although we do not explore this analytically, we do test it numerically in this section. After presenting the algorithm, we prove a convergence result based on the penalty parameter, and then give several numerical experiments verifying the theory and showing the effectiveness of the method.

As discussed in the introduction, the linear system of interest takes the form

$$
\left(\begin{array}{cc}
A & B \\
B^{T} & 0
\end{array}\right)\left(\begin{array}{l}
\hat{u} \\
\hat{p}
\end{array}\right)=\left(\begin{array}{c}
F \\
0
\end{array}\right)
$$

and arises from the finite element problem for the NS equations with homogeneous Dirichlet boundary conditions, which reads as follows.

Algorithm 3.1 (Pointwise divergence free solution for NS). Find $\left(u_{h}, P_{h}\right) \in\left(X_{h}, Q_{h}\right)$ satisfying

$$
\begin{aligned}
a\left(u_{h}, v_{h}\right)-\left(P_{h}, \nabla \cdot v_{h}\right) & =\left(f, v_{h}\right) \forall v_{h} \in X_{h}, \\
\left(\nabla \cdot u_{h}, q_{h}\right) & =0 \forall q_{h} \in Q_{h} .
\end{aligned}
$$

Remark 3.2. As discussed in section 2, with this definition of the bilinear form a, Algorithm 3.1 is relevant to both steady and unsteady problems. In the latter case, Algorithm 3.1 would be used at each timestep, with the right hand side function $f$ changing potentially each time step. 
Homogeneous Dirichlet boundary conditions are used here for simplicity, but extension to inhomogeneous Dirichlet boundary conditions or to partially Neumann boundary conditions can be performed with analogous arguments. We note that using grad-div stabilization in Algorithm 3.1 will not change the solution, since here divergence-free elements will provide that $\nabla \cdot u_{h}=0$ pointwise.

The Yosida method, which we call the Yosida-penalty method consists of approximating solutions of Algorithm 3.1 by replacing the system matrix with the inexact block LU approximation

$$
\left(\begin{array}{cc}
A & B \\
B^{T} & 0
\end{array}\right) \approx\left(\begin{array}{cc}
A & 0 \\
B^{T} & -\widetilde{S}
\end{array}\right)\left(\begin{array}{cc}
I & A^{-1} B \\
0 & I
\end{array}\right)
$$

where

- $A$ is the matrix arising from $a\left(u_{h}, v_{h}\right)+\gamma\left(\nabla \cdot u_{h}, \nabla \cdot v_{h}\right)$,

- $\widetilde{A}$ is the SPD matrix arising in the same manner as $A$, but without the nonlinear term, i.e. from

$$
a\left(u_{h}, v_{h}\right)+\gamma\left(\nabla \cdot u_{h}, \nabla \cdot v_{h}\right)-b^{*}\left(U, u_{h}, v_{h}\right),
$$

or equivalently, from

$$
\widetilde{a}\left(u_{h}, v_{h}\right)+\gamma\left(\nabla \cdot u_{h}, \nabla \cdot v_{h}\right),
$$

and using the same boundary conditions which create the matrix $A$. Often in Yosida methods, the viscous term is also omitted from $\widetilde{A}$, however to get $H^{1}$ bounds on the velocity error that are independent of $h$, we found it helpful to keep the viscous term.

- $B$ is the matrix arising from $-\left(p_{h}, \nabla \cdot v_{h}\right)$,

- $B^{T}$ is the matrix arising from $-\left(q_{h}, \nabla \cdot u_{h}\right)$.

Note that the construction of $\widetilde{A}$ is made together with the same boundary conditions used to construct $A$, and most importantly, $\widetilde{A}$ is SPD and therefore $\widetilde{S}$ is also. These matrix properties are the main advantage of Yosida methods, since we can use conjugate gradient methods for both the inner and outer solvers of the approximated Schur complement, which will provide for more efficient and robust solves.

Remark 3.3. Grad-div stabilization, originally developed in [10], and critically analyzed in [21], creates difficulties in solving with $A$ or $\widetilde{A}$ with larger $\gamma$, since the matrix that arises from graddiv stabilization is only symmetric positive semi-definite, with a non-trivial nullspace. However, effective solvers have recently been developed that can be successful in such cases, such as [29, 18], and see also the discussion in section 5.1 .4 in [22].

\subsection{Analysis of Yosida-penalty method}

We prove now that solutions of the Yosida-penalty method converge with rate $O\left(\gamma^{-2}\right)$ to the divergence free coupled method solution $\left(u_{h}, P_{h}\right)$ of Algorithm 3.1, in the natural norms of the finite element problems. 
A key first step in the analysis is the identification of the approximated linear algebraic system as finite element problems. Since the approximation is written as a block LU factorization, we can calculate

$$
\begin{aligned}
\left(\begin{array}{c}
\hat{u} \\
\hat{p}
\end{array}\right)=\mathbb{U}^{-1} \mathbb{L}^{-1}\left(\begin{array}{c}
F \\
0
\end{array}\right) & =\left(\begin{array}{cc}
I & A^{-1} B \\
0 & I
\end{array}\right)^{-1}\left(\begin{array}{cc}
A & 0 \\
B^{T} & -\widetilde{S}
\end{array}\right)^{-1}\left(\begin{array}{c}
F \\
0
\end{array}\right) \\
& =\left(\begin{array}{cc}
I & -A^{-1} B \\
0 & I
\end{array}\right)\left(\begin{array}{cc}
A^{-1} & 0 \\
\widetilde{S}^{-1} B^{T} A^{-1} & -\widetilde{S}^{-1}
\end{array}\right)\left(\begin{array}{c}
F \\
0
\end{array}\right) \\
& =\left(\begin{array}{cc}
I & -A^{-1} B \\
0 & I
\end{array}\right)\left(\begin{array}{c}
A^{-1} F \\
\widetilde{S}^{-1} B^{T} A^{-1} F
\end{array}\right) \\
& =\left(\begin{array}{c}
A^{-1} F-A^{-1} B \widetilde{S}^{-1} B^{T} A^{-1} F \\
\widetilde{S}^{-1} B^{T} A^{-1} F
\end{array}\right) .
\end{aligned}
$$

Thus, the problem reduces to three steps:

Step 1 : Solve $A \hat{z}=F$ for $\hat{z}$,

Step 2 : Solve $\widetilde{S} \hat{p}=B^{T} \hat{z}$ for $\hat{p}$,

Step 3 : Solve $A \hat{u}=A \hat{z}-B \hat{p}$ for $\hat{u}$.

Each of these steps can be reformulated into the following equivalent finite element problems:

Step 1 : Find $z_{h} \in X_{h}$ satisfying for all $v_{h} \in X_{h}$,

$$
a\left(z_{h}, v_{h}\right)+\gamma\left(\nabla \cdot z_{h}, \nabla \cdot v_{h}\right)=\left(f, v_{h}\right)
$$

Step 2 : Find $\left(\chi_{h}, p_{h}\right) \in\left(X_{h}, Q_{h}\right)$ satisfying for all $\left(v_{h}, r_{h}\right) \in\left(X_{h}, Q_{h}\right)$

$$
\begin{aligned}
\sigma\left(\chi_{h}, v_{h}\right)+\nu\left(\nabla \chi_{h}, \nabla v_{h}\right)+\gamma\left(\nabla \cdot \chi_{h}, \nabla \cdot v_{h}\right)-\left(p_{h}, \nabla \cdot v_{h}\right) & =0, \\
\left(\nabla \cdot \chi_{h}, r_{h}\right) & =-\left(\nabla \cdot z_{h}, r_{h}\right) .
\end{aligned}
$$

Step $3:$ Find $w_{h} \in X_{h}$ satisfying for all $v_{h} \in X_{h}$,

$$
a\left(w_{h}, v_{h}\right)+\gamma\left(\nabla \cdot w_{h}, \nabla \cdot v_{h}\right)=a\left(z_{h}, v_{h}\right)+\gamma\left(\nabla \cdot z_{h}, \nabla \cdot v_{h}\right)+\left(\nabla \cdot v_{h}, p_{h}\right) .
$$

We now prove a convergence result for the Yosida-penalty solution to the solution of Algorithm 3.1 , based on the penalty parameter $\gamma$. Thus, in terms of solutions in the finite element spaces, the Yosida-penalty solutions are $\left(w_{h}, p_{h}-\gamma \nabla \cdot w_{h}\right) \in\left(X_{h}, Q_{h}\right)$, as the convergence result reveals.

Theorem 3.1. Let $\left(u_{h}, P_{h}\right) \in\left(X_{h}, Q_{h}\right)$ be the coupled method solution of Algorithm 3.1, and $\left(w_{h}, p_{h}\right) \in\left(X_{h}, Q_{h}\right)$ be the Yosida method solution from (3.5)-(3.8) with parameter $\gamma \geq 1$. Denoting $\hat{p}_{h}:=p_{h}-\gamma \nabla \cdot w_{h}$, the following bound holds with $C$ a data dependent constant,

$$
\left\|\nabla\left(w_{h}-u_{h}\right)\right\|+\left\|P_{h}-\hat{p}_{h}\right\| \leq C \gamma^{-2} .
$$


Proof. The proof is split into 3 parts, which analyze the individual steps of the Yosida method. We note that since $P_{h}$ is the pressure solution of the coupled problem, it is straightforward to show that $\left\|P_{h}\right\| \leq C_{P}:=\beta^{-1}\|f\|_{X_{h}^{\prime}}\left(\left(\sigma \lambda^{2}+\nu+C_{s} C_{U}\right) \nu^{-1}+1\right)$.

Proof Step 1. Claim: $a\left(z_{h}, v_{h}\right)=a\left(u_{h}, v_{h}\right) \forall v_{h} \in V_{h}$, and $\left\|\nabla \cdot z_{h}\right\| \leq \gamma^{-1} C_{P}$.

To analyze Step 1, we begin by subtracting (3.2) from (3.5). This gives

$$
a\left(z_{h}-u_{h}, v_{h}\right)+\gamma\left(\nabla \cdot z_{h}, \nabla \cdot v_{h}\right)=-\left(P_{h}, \nabla \cdot v_{h}\right) \forall v_{h} \in X_{h} .
$$

We note that restricting $v_{h}$ to $V_{h}$ yields the first stated claim. For the second claim, choose $v_{h}=z_{h}-u_{h}$ in (3.10). Noting $\nabla \cdot u_{h}=0$ and applying Cauchy-Schwarz and Young's inequalities yields

$$
\left\|z_{h}-u_{h}\right\|_{\tilde{a}}^{2}+\gamma\left\|\nabla \cdot z_{h}\right\|^{2}=-\left(P_{h}, \nabla \cdot z_{h}\right) \leq \frac{\gamma}{2}\left\|\nabla \cdot z_{h}\right\|^{2}+\frac{\gamma^{-1}}{2}\left\|P_{h}\right\|^{2} .
$$

Reducing and using the bound on $\left\|P_{h}\right\|$ produces the second stated claim.

Proof Step 2. Claim: $\left\|p_{h}+\gamma \nabla \cdot z_{h}\right\| \leq \gamma^{-1} C_{P} C_{\tilde{a}} \beta^{-1}$, and $\left\|p_{h}\right\| \leq\left(\gamma^{-1} C_{\tilde{a}} \beta^{-1}+1\right) C_{P}$.

For the step 2 analysis, begin by setting $r_{h}=\nabla \cdot\left(\chi_{h}+z_{h}\right)$ in (3.7), which yields

$$
\left\|\nabla \cdot\left(z_{h}+\chi_{h}\right)\right\|=0 \text {. }
$$

Thus we can rewrite $(3.6)$ as

$$
\widetilde{a}\left(\chi_{h}, v_{h}\right)-\gamma\left(\nabla \cdot z_{h}, \nabla \cdot v_{h}\right)-\left(p_{h}, \nabla \cdot v_{h}\right)=0 .
$$

The choice of $v_{h}=\chi_{h}^{0} \in V_{h}$ gives immediately that $\left\|\chi_{h}^{0}\right\|_{\tilde{a}}=0$, and thus that $\chi_{h} \in R_{h}^{\tilde{a}}$. Using this, dividing both sides of (3.12) by $\left\|v_{h}\right\|_{\tilde{a}}$, applying the inf-sup condition and (2.3) yields

$$
\beta\left\|p_{h}+\gamma \nabla \cdot z_{h}\right\| \leq \frac{\left|\widetilde{a}\left(\chi_{h}, v_{h}\right)\right|}{\left\|v_{h}\right\|_{\tilde{a}}} \leq\left\|\chi_{h}\right\|_{\tilde{a}} \leq C_{\tilde{a}}\left\|\nabla \cdot \chi_{h}\right\| .
$$

Thanks to this, (3.11), and claim 2 of step 1, we get

$$
\begin{aligned}
\left\|p_{h}+\gamma \nabla \cdot z_{h}\right\| & \leq C_{\tilde{a}} \beta^{-1}\left\|\nabla \cdot \chi_{h}\right\| \\
& =C_{\tilde{a}} \beta^{-1}\left\|\nabla \cdot z_{h}\right\| \\
& \leq \gamma^{-1} C_{P} C_{\tilde{a}} \beta^{-1} .
\end{aligned}
$$

This proves the first stated claim. For the second, apply the triangle inequality to (3.13) to find that

$$
\left\|p_{h}\right\| \leq\left\|p_{h}+\gamma \nabla \cdot z_{h}\right\|+\gamma\left\|\nabla \cdot z_{h}\right\| \leq\left(\gamma^{-1} C_{\tilde{a}} \beta^{-1}+1\right) C_{P}
$$

Proof Step 3. (completes the proof for velocity)

We begin the step 3 analysis by bounding $w_{h}-z_{h}$, and thus we start by rewriting (3.8) in terms of $w_{h}-z_{h}$ :

$$
a\left(w_{h}-z_{h}, v_{h}\right)+\gamma\left(\nabla \cdot\left(w_{h}-z_{h}\right), \nabla \cdot v_{h}\right)=\left(p_{h}, \nabla \cdot v_{h}\right) .
$$

Choosing $v_{h}=w_{h}-z_{h}$ immediately produces the bound $\left\|\nabla \cdot\left(w_{h}-z_{h}\right)\right\| \leq \gamma^{-1}\left\|p_{h}\right\|$. Orthogonally decompose $w_{h}$ and $z_{h}$ into pieces in and out of $V_{h}$ using the $\widetilde{a}$ inner product, and write

$$
\left(w_{h}-z_{h}\right)=\left(w_{h}^{0}-z_{h}^{0}\right)+\left(w_{h}^{r}-z_{h}^{r}\right)
$$


where $w_{h}^{r}, z_{h}^{r} \in R_{h}^{\tilde{a}}$ and $w_{h}^{0}, z_{h}^{0} \in V_{h}$. By Lemma 2.2, we have established

$$
\left\|w_{h}^{r}-z_{h}^{r}\right\|_{\tilde{a}} \leq C_{\tilde{a}}\left\|\nabla \cdot\left(w_{h}^{r}-z_{h}^{r}\right)\right\|=C_{\tilde{a}}\left\|\nabla \cdot\left(w_{h}-z_{h}\right)\right\| \leq \gamma^{-1} C_{\tilde{a}}\left\|p_{h}\right\| \leq \gamma^{-1} C_{\tilde{a}}\left(\gamma^{-1} C_{\tilde{a}} \beta^{-1}+1\right) C_{P} .
$$

To bound the remaining part of $w_{h}-z_{h}$, choose $v_{h}=w_{h}^{0}-z_{h}^{0}$ in (3.14). Using $\widetilde{a}$-orthogonality and skew symmetry of $b^{*}$, we have that

$$
\begin{aligned}
\left\|w_{h}^{0}-z_{h}^{0}\right\|_{\tilde{a}}^{2}=-b^{*}\left(U, w_{h}-z_{h}, w_{h}^{0}-z_{h}^{0}\right) & =-b^{*}\left(U, w_{h}^{r}-z_{h}^{r}, w_{h}^{0}-z_{h}^{0}\right) \\
& \leq C_{s}\|\nabla U\|\left\|\nabla\left(w_{h}^{r}-z_{h}^{r}\right)\right\|\left\|\nabla\left(w_{h}^{0}-z_{h}^{0}\right)\right\| \\
& \leq C_{s} C_{U} \nu^{-1}\left\|w_{h}^{r}-z_{h}^{r}\right\| \tilde{a}\left\|w_{h}^{0}-z_{h}^{0}\right\|_{\tilde{a}},
\end{aligned}
$$

which reduces to

$$
\left\|w_{h}^{0}-z_{h}^{0}\right\|_{\tilde{a}} \leq C_{s} C_{U} \nu^{-1}\left\|w_{h}^{r}-z_{h}^{r}\right\|_{\tilde{a}} .
$$

Thus, combining the bounds on the parts of $w_{h}-z_{h}$,

$$
\left\|w_{h}-z_{h}\right\|_{\tilde{a}}=\left(1+C_{s} C_{U} \nu^{-1}\right)\left\|w_{h}^{r}-z_{h}^{r}\right\|_{\tilde{a}} \leq \gamma^{-1} C_{\tilde{a}}\left(1+C_{s} C_{U} \nu^{-1}\right)\left(\gamma^{-1} C_{\tilde{a}} \beta^{-1}+1\right) C_{P} \leq K \gamma^{-1},
$$

with $K=C_{\tilde{a}}\left(1+C_{s} C_{U} \nu^{-1}\right)\left(C_{\tilde{a}} \beta^{-1}+1\right) C_{P}$, and using the assumption $\gamma \geq 1$.

Next, take $v_{h}=w_{h}^{r}$ in (3.8) and apply Cauchy-Schwarz along with Lemma 2.2 to get

$$
\begin{aligned}
\left\|w_{h}^{r}\right\|_{\tilde{a}}^{2}+\gamma\left\|\nabla \cdot w_{h}^{r}\right\|^{2} & =\widetilde{a}\left(z_{h}^{r}, w_{h}^{r}\right)-b^{*}\left(U, w_{h}-z_{h}, w_{h}^{r}\right)+\left(\gamma \nabla \cdot z_{h}^{r}+p_{h}, \nabla \cdot w_{h}^{r}\right) \\
& \leq\left\|z_{h}^{r}\right\|_{\tilde{a}}\left\|w_{h}^{r}\right\|_{\tilde{a}}+C_{s}\|\nabla U\|\left\|\nabla\left(w_{h}-z_{h}\right)\right\|\left\|\nabla w_{h}^{r}\right\|+\left\|\gamma \nabla \cdot z_{h}^{r}+p_{h}\right\|\left\|\nabla \cdot w_{h}^{r}\right\| \\
& \leq\left(C_{\tilde{a}}^{2}\left\|\nabla \cdot z_{h}^{r}\right\|+C_{s} C_{\tilde{a}} \nu^{-1} C_{U}\left\|w_{h}-z_{h}\right\|_{\tilde{a}}+\left\|\gamma \nabla \cdot z_{h}^{r}+p_{h}\right\|\right)\left\|\nabla \cdot w_{h}^{r}\right\| .
\end{aligned}
$$

This reduces with Young's inequality to

$$
\left\|\nabla \cdot w_{h}^{r}\right\| \leq \gamma^{-1}\left(C_{\tilde{a}}^{2}\left\|\nabla \cdot z_{h}^{r}\right\|+C_{s} C_{\tilde{a}} \nu^{-1} C_{U}\left\|w_{h}-z_{h}\right\|_{\tilde{a}}+\left\|\gamma \nabla \cdot z_{h}^{r}+p_{h}\right\|\right),
$$

and now applying the results from steps 1 and 2, and (3.16), we obtain the bound

$$
\left\|\nabla \cdot w_{h}^{r}\right\| \leq \gamma^{-2}\left(C_{s} C_{\tilde{a}} \nu^{-1} C_{U} K+\left(C_{\tilde{a}}^{2}+C_{\tilde{a}} \beta^{-1}\right) C_{P}\right),
$$

Since $u_{h} \in V_{h}, u_{h}^{r}=0$, and we get that

$$
\left\|w_{h}^{r}-u_{h}^{r}\right\|_{\tilde{a}}=\left\|w_{h}^{r}\right\|_{\tilde{a}} \leq C_{\tilde{a}}\left\|\nabla \cdot w_{h}^{r}\right\| \leq \gamma^{-2} C_{\tilde{a}}^{2}\left(C_{s} \beta^{-1} \nu^{-1} C_{U} K+C_{\tilde{a}} C_{P}+\beta^{-1} C_{P}\right) .
$$

It remains to bound $\left\|w_{h}^{0}-u_{h}^{0}\right\|_{\tilde{a}}$. From (3.8), choosing $v_{h}=w_{h}^{0}-u_{h}^{0}$, we have

$$
a\left(w_{h}-u_{h}, w_{h}^{0}-u_{h}^{0}\right)=a\left(z_{h}-u_{h}, w_{h}^{0}-u_{h}^{0}\right),
$$

but from step 1 (claim 1 ), the term on the right side vanishes since $w_{h}^{0}-u_{h}^{0} \in V_{h}$. Decomposing the bilinear form $a$ of the remaining term and using skew-symmetry of $b^{*}$, we obtain

$$
\begin{aligned}
\left\|w_{h}^{0}-u_{h}^{0}\right\|_{\tilde{a}}^{2} & =-b^{*}\left(U, w_{h}-u_{h}, w_{h}^{0}-u_{h}^{0}\right) \\
& =-b^{*}\left(U, w_{h}^{r}-u_{h}^{r}, w_{h}^{0}-u_{h}^{0}\right) \\
& \leq C_{s} \nu^{-1} C_{U}\left\|w_{h}^{r}-u_{h}^{r}\right\|_{\tilde{a}}\left\|w_{h}^{0}-u_{h}^{0}\right\|_{\tilde{a}},
\end{aligned}
$$


which simplifies to

$$
\left\|w_{h}^{0}-u_{h}^{0}\right\|_{\tilde{a}} \leq C_{s} C_{U} \nu^{-1}\left\|w_{h}^{r}-u_{h}^{r}\right\|_{\tilde{a}} .
$$

Hence we have bounded $\left\|w_{h}^{0}-u_{h}^{0}\right\|_{\tilde{a}}$ and $\left\|w_{h}^{r}-u_{h}^{r}\right\|_{\tilde{a}}$, and we can conclude that

$$
\begin{aligned}
\left\|\nabla\left(w_{h}-u_{h}\right)\right\| & \leq \nu^{-1 / 2}\left\|w_{h}-u_{h}\right\|_{\tilde{a}} \\
& =\nu^{-1 / 2}\left(\left\|w_{h}^{0}-u_{h}^{0}\right\|_{\tilde{a}}+\left\|w_{h}^{r}-u_{h}^{r}\right\|_{\tilde{a}}\right) \\
& \leq \nu^{-1 / 2}\left(1+C_{s} C_{U} \nu^{-1}\right)\left\|w_{h}^{r}-u_{h}^{r}\right\|_{\tilde{a}} \\
& \leq \gamma^{-2} \nu^{-1 / 2} C_{\tilde{a}}^{2}\left(1+C_{s} C_{U} \nu^{-1}\right)\left(C_{s} \beta^{-1} \nu^{-1} C_{U} K+C_{\tilde{a}} C_{P}+\beta^{-1} C_{P}\right) .
\end{aligned}
$$

\section{Proof of pressure convergence}

For pressure convergence, combining (3.5) and (3.8) produces

$$
a\left(w_{h}, v_{h}\right)+\left(\gamma \nabla \cdot w_{h}-p_{h}, \nabla \cdot v_{h}\right)=\left(f, v_{h}\right) \forall v_{h} \in X_{h} .
$$

Denoting $\hat{p}_{h}=p_{h}-\gamma \nabla \cdot w_{h}$ and subtracting (3.18) from (3.2) yields

$$
\left(P_{h}-\hat{p}_{h}, \nabla \cdot v_{h}\right)=a\left(u_{h}-w_{h}, v_{h}\right) \forall v_{h} \in X_{h} .
$$

Majorizing the right hand side using similar analysis as above, then applying inf-sup produces the estimate

$$
\beta\left\|P_{h}-\hat{p}_{h}\right\| \leq C_{\tilde{a}}\left\|u_{h}-w_{h}\right\|_{\tilde{a}},
$$

and from here we use the velocity convergence bound for $\left\|u_{h}-w_{h}\right\|_{\tilde{a}}$.

\subsection{Numerical experiments}

In this section we provide several numerical experiments to test both the theory and usefulness of the Yosida-penalty method. For our 2D tests, we use $\left(P_{2}, P_{1}^{\text {disc }}\right)$ Scott-Vogelius (SV) elements on barycenter refined triangular meshes, which is known to be inf-sup stable [1]. In $3 \mathrm{D}$, we use $\left(P_{3}, P_{2}^{\text {disc }}\right) \mathrm{SV}$ elements on barycenter refined tetrahedral meshes, which is known from [33] to be inf-sup stable.

Each of our four tests shows second order convergence of the Yosida-penalty solutions as $\gamma \rightarrow \infty$ to the SV solution. The first two tests are for an analytic test problem with known solution, and are used to verify the predicted convergence rate of 2 to the SV solution, but also to compare the accuracy of the Yosida-penalty and pressure-corrected Yosida-penalty solutions. The final two tests are for physically meaningful problems: channel flow over a step, and the 3D lid-driven cavity problem. All of our tests indicate that the Yosida-penalty method with $\gamma \geq 10$ can have accuracy very close to that of Algorithm 3.1 when no approximations are made to the linear system.

To solve the linear algebraic systems, a direct solver was used to solve with $A$ and $\widetilde{A}$, including the inner solves of the Schur complements. The outer solvers for the Schur complements used were conjugate gradient (CG) for $\widetilde{S}$ and BICGSTAB for $S$, with tolerance $1 e-10$, and with the pressure mass matrix as a preconditioner. We note that since the pressure space is discontinuous, the full pressure mass matrix was used (instead of a diagonally lumped) since it is block diagonal with $3 \times 3$ blocks in $2 \mathrm{D}$ and $10 \times 10$ blocks in 3D. In all cases, for $\gamma \geq 1,000$ the number of CG iterations 2 or 3 , for $10 \leq \gamma \leq 100$ it was 4 or 5 , and for $\gamma=1$, the number ranged from 5 to 15 . 


\subsubsection{Numerical experiment 1: Convergence rate verification}

Our first numerical test is for the verification of the predicted convergence rates of Theorem 3.1. To do this, we chose an analytical test problem on $\Omega=(0,1)^{2}$ with solution

$$
u=\left(\begin{array}{c}
\cos (y) \\
\sin (x)
\end{array}\right), p=\sin (x),
$$

and calculated $f$ using

$$
f=\sigma u+U \cdot \nabla u+\nabla p-\nu \Delta u
$$

with $U=u, \sigma=1$, and $\nu=1$. The discretization used Algorithm 3.1 with $\left(P_{2}, P_{1}^{\text {disc }}\right) \mathrm{SV}$ elements to build the linear systems, and then solved the linear systems using the Yosida-penalty method (inexact block LU factorization (3.4)) using several choices of $\gamma$. A $h=1 / 32$ barycenter refined uniform mesh was used, which is known to provide inf-sup stability for this element choice [1]. The inhomogeneous Dirichlet boundary condition was enforced nodally using the true solution.

Results of the computations are shown in table 1, as the differences between the SV solution and Yosida-penalty method solutions, in the natural norms of the problem. We observe the theorypredicted convergence rate of 2 for both velocity and pressure, and the error (to the true solution, shown in last column) is seen to decrease by about 2 orders of magnitude if $\gamma$ is increased from $\gamma=0$ to $\gamma \geq 10$; even with $\gamma=1$ a significant reduction in error is observed.

\begin{tabular}{|c|c|c|c|c|c|c|c|}
\hline$\gamma$ & $\left\|\nabla\left(u_{Y}^{\gamma}-u_{S V}\right)\right\|$ & rate & $\left\|\nabla \cdot u_{Y}^{\gamma}\right\|$ & rate & $\left\|\hat{p}_{Y}^{\gamma}-p_{S V}\right\|$ & rate & $\left\|\nabla\left(u_{Y}^{\gamma}-u_{\text {true }}\right)\right\|$ \\
\hline 0 & $1.057 \mathrm{e}-02$ & - & $9.218 \mathrm{e}-03$ & - & $1.352 \mathrm{e}-2$ & - & $1.057 \mathrm{e}-02$ \\
\hline 0.1 & $9.417 \mathrm{e}-03$ & - & $8.146 \mathrm{e}-03$ & - & $1.218 \mathrm{e}-2$ & - & $9.418 \mathrm{e}-03$ \\
\hline 1 & $4.369 \mathrm{e}-03$ & 0.33 & $3.598 \mathrm{e}-03$ & 0.36 & $6.038 \mathrm{e}-3$ & 0.31 & $4.371 \mathrm{e}-03$ \\
\hline 10 & $3.028 \mathrm{e}-04$ & 1.16 & $2.259 \mathrm{e}-04$ & 1.20 & $4.802 \mathrm{e}-4$ & 1.10 & $3.360 \mathrm{e}-04$ \\
\hline 100 & $4.629 \mathrm{e}-06$ & 1.82 & $3.296 \mathrm{e}-06$ & 1.84 & $7.971 \mathrm{e}-6$ & 1.78 & $1.456 \mathrm{e}-04$ \\
\hline 1,000 & $4.878 \mathrm{e}-08$ & 1.98 & $3.446 \mathrm{e}-08$ & 1.98 & $8.537 \mathrm{e}-8$ & 1.97 & $1.455 \mathrm{e}-04$ \\
\hline 10,000 & $5.303 \mathrm{e}-10$ & 1.96 & $3.462 \mathrm{e}-10$ & 2.00 & $1.920 \mathrm{e}-9$ & 1.65 & $1.455 \mathrm{e}-04$ \\
\hline
\end{tabular}

Table 1: Differences between the SV solution and the Yosida-penalty solutions with varying $\gamma$, as well as the error in the Yosida-penalty method solutions.

\subsubsection{Numerical experiment 2: Comparison of Yosida-penalty and pressure-correction Yosida-penalty}

Our next experiment compares the accuracy of the Yosida-penalty method with the pressurecorrected Yosida-penalty method of [28]. To do this, we repeat the same experiment as above in numerical experiment 1 , but we alter the inexact block LU factorization to be instead

$$
\left(\begin{array}{cc}
A & B \\
B^{T} & 0
\end{array}\right) \approx\left(\begin{array}{cc}
A & 0 \\
B^{T} & -B^{T} \widetilde{A}^{-1} B
\end{array}\right)\left(\begin{array}{cc}
I & A^{-1} B \\
0 & Q
\end{array}\right)
$$

with $Q=\left(B^{T} \widetilde{A}^{-1} A \widetilde{A}^{-1} B\right)^{-1} S$. This factorization was proven in [28] to be $O\left(\sigma^{-3}\right)$ accurate in the velocity, instead of $O\left(\sigma^{-2}\right)$ as it is when $Q=I$. This gain in accuracy comes at about double the cost, as it requires us to perform an additional solve with the SPD approximated Schur complement, and two more solves with the SPD matrix $\widetilde{A}$. 
The results are shown in table 2. As $\gamma$ increases, we observe the convergence rate of 2 to the $\mathrm{SV}$ solution (noting the reduction in rate for $\gamma=10,000$ is due to already reaching the tolerance of the linear solvers. Hence, we observe there is no improvement in convergence rate as $\gamma$ increases for the pressure-corrected Yosida-penalty method, compared to the Yosida-penalty method. However, we do observe by comparing the error in solutions from tables 1 and 2 that for smaller values of $\gamma$, the pressure-corrected method is significantly more accurate, while for $\gamma \geq 10$, the accuracy of the methods in similar; this is expected due to the increased accuracy with respect to $\sigma$. A significant increase in accuracy is still observed in the $\gamma \geq 10$ solution compared to the $\gamma=0$ solution in the pressure-corrected Yosida-penalty method.

\begin{tabular}{|c|c|c|c|c|c|c|c|}
\hline$\gamma$ & $\left\|\nabla\left(u_{Y}^{\gamma}-u_{S V}\right)\right\|$ & rate & $\left\|\nabla \cdot u_{Y}^{\gamma}\right\|$ & rate & $\left\|\hat{p}_{Y}^{\gamma}-p_{S V}\right\|$ & rate & $\left\|\nabla\left(u_{Y}^{\gamma}-u_{\text {true }}\right)\right\|$ \\
\hline 0 & $2.156 \mathrm{e}-04$ & - & $1.378 \mathrm{e}-04$ & - & $3.783 \mathrm{e}-4$ & - & $2.603 \mathrm{e}-04$ \\
\hline 0.1 & $2.000 \mathrm{e}-04$ & - & $1.268 \mathrm{e}-04$ & - & $3.350 \mathrm{e}-4$ & - & $2.474 \mathrm{e}-04$ \\
\hline 1 & $1.161 \mathrm{e}-04$ & 0.24 & $7.031 \mathrm{e}-05$ & 0.26 & $2.119 \mathrm{e}-4$ & 0.22 & $1.862 \mathrm{e}-04$ \\
\hline 10 & $1.165 \mathrm{e}-05$ & 1.00 & $6.535 \mathrm{e}-06$ & 1.03 & $2.669 \mathrm{e}-5$ & 0.90 & $1.460 \mathrm{e}-04$ \\
\hline 100 & $1.994 \mathrm{e}-07$ & 1.77 & $1.085 \mathrm{e}-07$ & 1.78 & $4.020 \mathrm{e}-7$ & 1.82 & $1.455 \mathrm{e}-04$ \\
\hline 1,000 & $2.130 \mathrm{e}-09$ & 1.97 & $1.153 \mathrm{e}-09$ & 1.97 & $4.319 \mathrm{e}-9$ & 1.97 & $1.455 \mathrm{e}-04$ \\
\hline 10,000 & $2.016 \mathrm{e}-10$ & 1.02 & $1.160 \mathrm{e}-11$ & 2.00 & $1.321 \mathrm{e}-9$ & 0.51 & $1.455 \mathrm{e}-04$ \\
\hline
\end{tabular}

Table 2: Differences between the SV solution and the pressure corrected Yosida-penalty solutions with varying $\gamma$, as well as the error in the Yosida-penalty method solutions.

\subsubsection{Numerical experiment 3: 2D channel flow over a step}

We next test the Yosida-penalty scheme on a 2D channel flow problem past a step. The domain is a $40 \times 10$ rectangle, with a $1 \times 1$ step placed five units in from the left, at the bottom of the channel. The viscosity is set as $\nu=\frac{1}{20}$, and boundary conditions are enforced as no slip on the top and bottom walls, and along the step. At the inflow and outflow, a parabolic profile is enforced nodally to be $\left.u\right|_{\text {in/out }}$. We take $\sigma=0$, and $U$ to satisfy the same boundary conditions as $u$, but in the interior satisfy $U(x, y)=\left\langle\frac{y(10-y)}{25}, 0\right\rangle^{T}$.

Solutions were computed using SV elements, and with the Yosida-penalty method with several choices of $\gamma$. The differences between the SV solution and Yosida-penalty solutions are shown in table 3, and we again observe second order convergence, as predicted by the theory. Figure 1 shows plots of the velocity solutions of the schemes, as streamlines over speed contours, and the convergence to the SV solution as $\gamma$ increases is clear. Furthermore, we observe that smaller $\gamma(=0$, 0.1 , and 1) solutions do not predict the eddy behind the step, while the SV and larger $\gamma(\geq 10)$ do predict it. The smaller $\gamma$ solutions also do not accurately predict the speed contours, and also have oscillations at the outflow, due to the inaccuracy of the approximation of the Yosida-penalty method with small penalty parameter for steady problems. 
$\gamma=0$

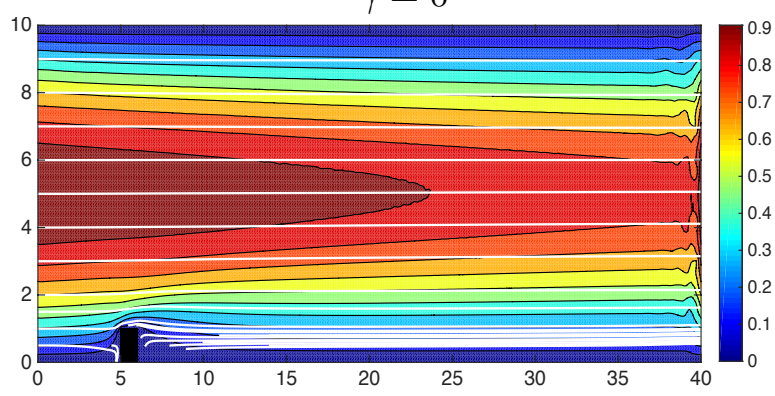

$\gamma=1$

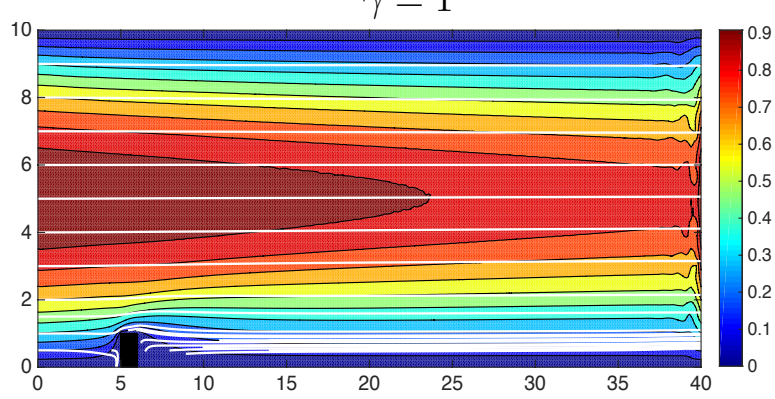

$\gamma=100$

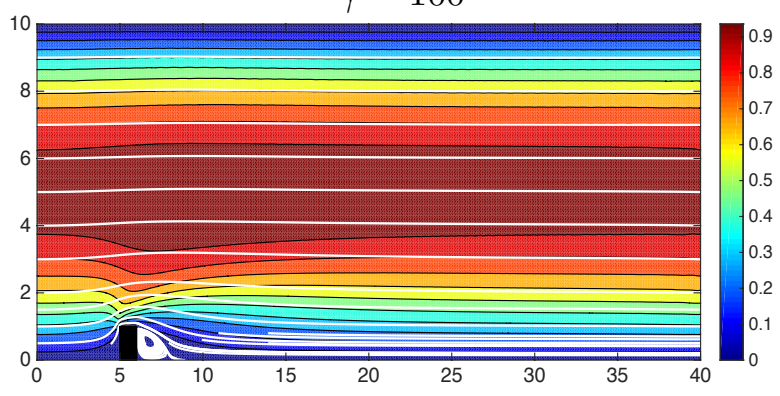

$\gamma=0.1$

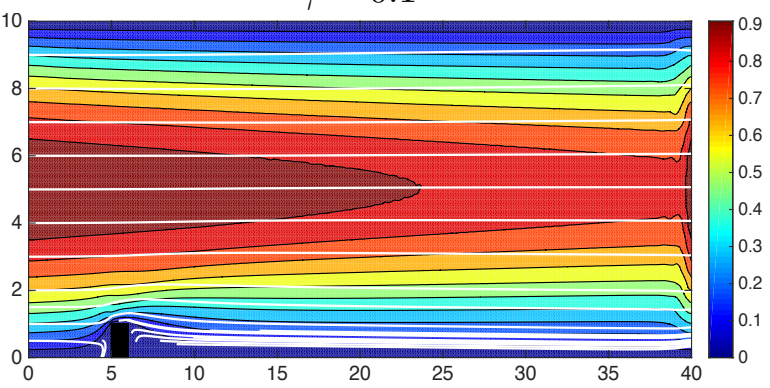

$\gamma=10$

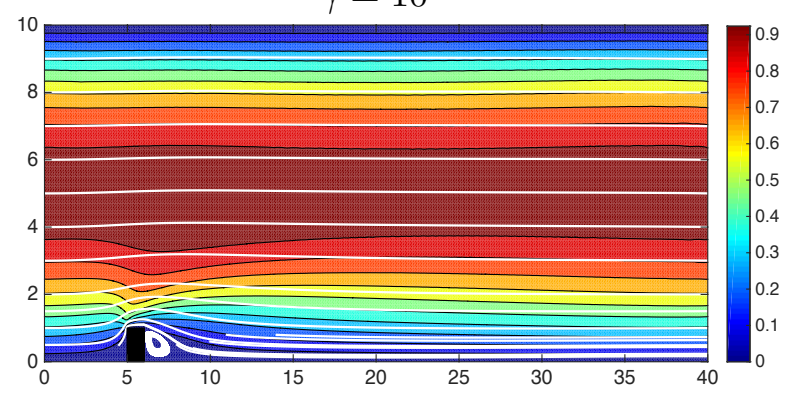

SV

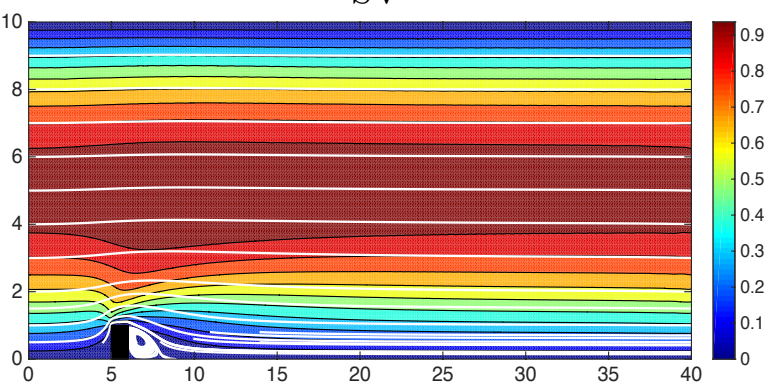

Figure 1: Shown above are plots of velocity streamlines over speed contours for varying $\gamma$ Yosidapenalty and SV solutions.

\begin{tabular}{|c|c|c|c|c|c|c|}
\hline$\gamma$ & $\left\|\nabla\left(u_{Y}^{\gamma}-u_{S V}\right)\right\|$ & rate & $\left\|\nabla \cdot u_{Y}^{\gamma}\right\|$ & rate & $\left\|\hat{p}_{Y}^{\gamma}-p_{S V}\right\|$ & rate \\
\hline 0 & $1.274 \mathrm{e}+00$ & - & $8.726 \mathrm{e}-01$ & - & $8.056 \mathrm{e}-1$ & - \\
\hline 0.1 & $1.026 \mathrm{e}+00$ & - & $5.214 \mathrm{e}-01$ & - & $7.844 \mathrm{e}-1$ & - \\
\hline 1 & $8.110 \mathrm{e}-01$ & 0.10 & $1.828 \mathrm{e}-01$ & 0.46 & $6.391 \mathrm{e}-1$ & 0.09 \\
\hline 10 & $1.440 \mathrm{e}-01$ & 0.75 & $2.466 \mathrm{e}-02$ & 0.87 & $1.143 \mathrm{e}-1$ & 0.75 \\
\hline 100 & $1.834 \mathrm{e}-03$ & 1.90 & $3.852 \mathrm{e}-04$ & 1.81 & $1.846 \mathrm{e}-3$ & 1.79 \\
\hline 1,000 & $1.854 \mathrm{e}-05$ & 2.00 & $3.967 \mathrm{e}-06$ & 1.99 & $1.928 \mathrm{e}-5$ & 1.98 \\
\hline 10,000 & $1.859 \mathrm{e}-07$ & 2.00 & $3.978 \mathrm{e}-08$ & 2.00 & $1.970 \mathrm{e}-7$ & 1.99 \\
\hline
\end{tabular}

Table 3: Differences between the SV solution and pressure-corrected Yosida-penalty solutions with varying $\gamma$ for the flow over a step benchmark problem. A convergence rate of 2 is observed, as predicted by the theory. 


\subsubsection{Numerical experiment 4: 3D lid driven cavity}

Our final test in this section is for the 3D lid driven cavity benchmark problem for steady NavierStokes equations. This is a widely used test problem, see e.g. [31] and references therein for more detailed description and history. The domain is the unit cube, and the problem uses no forcing $f=0$, homogeneous Dirichlet boundary conditions on the bottom and side walls, and on the lid $(z=1)$, the Dirichlet condition $u_{\text {lid }}=\langle 1,0,0\rangle^{T}$ is enforced. We consider the 3 benchmark tests of $R e=100,400$ and 1,000 (with $R e:=\nu^{-1}$ for this problem).

The problem is solved using a quasi-Newton method to resolve the nonlinearity, and each linear solves was done using the Yosida-penalty method, with the same $\gamma$ being used for every solve in the nonlinear iteration. We computed using $\left(P_{3}, P_{2}^{\text {disc }}\right) \mathrm{SV}$ elements on a barycenter refined mesh that provided 796,722 total degrees of freedom (477,282 velocity and 319,440 pressure). Solutions from each choice of $\gamma$ were compared to the SV solution, and the differences are shown in table 4. Again we observe the theory-predicted second order convergence rate. Plots of the midsliceplanes and of the centerline $\mathrm{x}$-velocities are shown in figures 2-3 for the $\gamma=1$ solution, and are in excellent agreement with DNS results of Wong and Baker [31], which used a similar number of degrees of freedom. We note that $\gamma=10,100$ and 1,000 gave identical plots (not shown).

\begin{tabular}{|c|c|c|c|c|c|c|}
\hline $\operatorname{Re}$ & $\gamma$ & dof & $\left\|u_{S V}-u_{Y}^{\gamma}\right\|$ & rate & $\left\|\nabla \cdot u_{Y}^{\gamma}\right\|$ & rate \\
\hline 100 & 1 & 796,722 & $1.504 \mathrm{e}-3$ & - & $4.370 \mathrm{e}-3$ & - \\
\hline 100 & 10 & 796,722 & $1.733 \mathrm{e}-5$ & 1.94 & $5.021 \mathrm{e}-5$ & 1.94 \\
\hline 100 & 100 & 796,722 & $1.758 \mathrm{e}-7$ & 1.99 & $5.092 \mathrm{e}-7$ & 1.99 \\
\hline 100 & 1,000 & 796,722 & $1.757 \mathrm{e}-9$ & 2.00 & $5.099 \mathrm{e}-9$ & 2.00 \\
\hline \hline 400 & 1 & 796,722 & $1.960 \mathrm{e}-3$ & - & $3.009 \mathrm{e}-3$ & - \\
\hline 400 & 10 & 796,722 & $2.487 \mathrm{e}-5$ & 1.90 & $3.820 \mathrm{e}-5$ & 1.90 \\
\hline 400 & 100 & 796,722 & $2.544 \mathrm{e}-7$ & 1.99 & $3.914 \mathrm{e}-7$ & 1.99 \\
\hline 400 & 1,000 & 796,722 & $2.524 \mathrm{e}-9$ & 2.00 & $3.923 \mathrm{e}-9$ & 2.00 \\
\hline \hline 1,000 & 1 & 796,722 & $2.382 \mathrm{e}-3$ & - & $2.233 \mathrm{e}-3$ & - \\
\hline 1,000 & 10 & 796,722 & $3.275 \mathrm{e}-5$ & 1.86 & $3.014 \mathrm{e}-5$ & 1.87 \\
\hline 1,000 & 100 & 796,722 & $3.377 \mathrm{e}-7$ & 1.99 & $3.108 \mathrm{e}-7$ & 1.99 \\
\hline 1,000 & 1,000 & 796,722 & $3.382 \mathrm{e}-9$ & 2.00 & $3.118 \mathrm{e}-9$ & 2.00 \\
\hline
\end{tabular}

Table 4: Difference to the SV solution, and convergence rates, for the Yosida-penalty method solutions of the 3D driven cavity tests.

\section{Connection to the classical iterated penalty method}

In the previous section, we proved a convergence result for the Yosida-penalty method solution to the solution of the coupled system, i.e. the solution resulting from solving the exact algebraic system. The proof was based on transforming the inexact block LU factorization into finite element problems, and analyzing the resulting sub-problems. From the analysis, there was a clear connection to iterated penalty methods, which solve similar problems repeatedly in an iteration that also converges to the coupled method solution. In this sense, the Yosida-penalty method consists of two iterated-penalty type steps and one approximated SPD approximated Schur complement solve. 

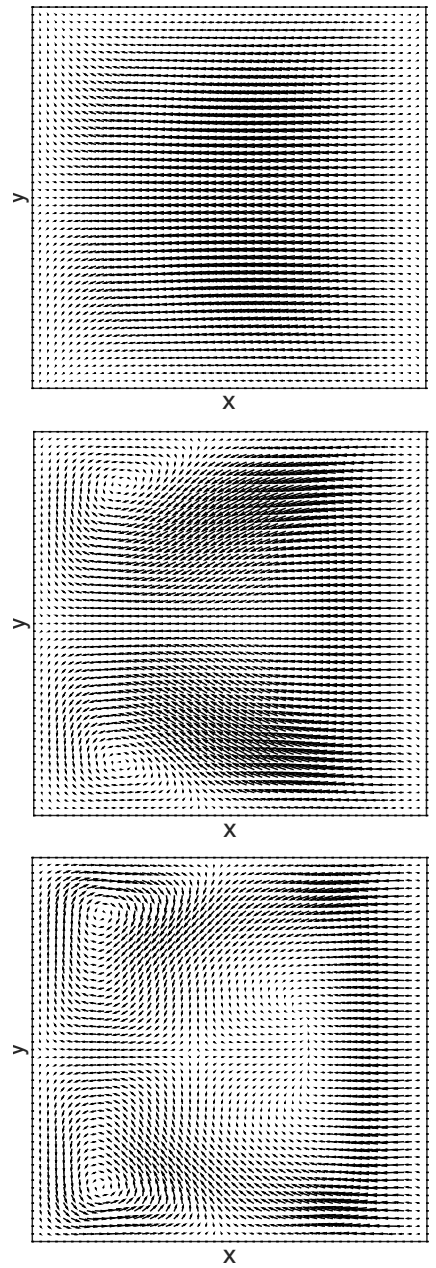
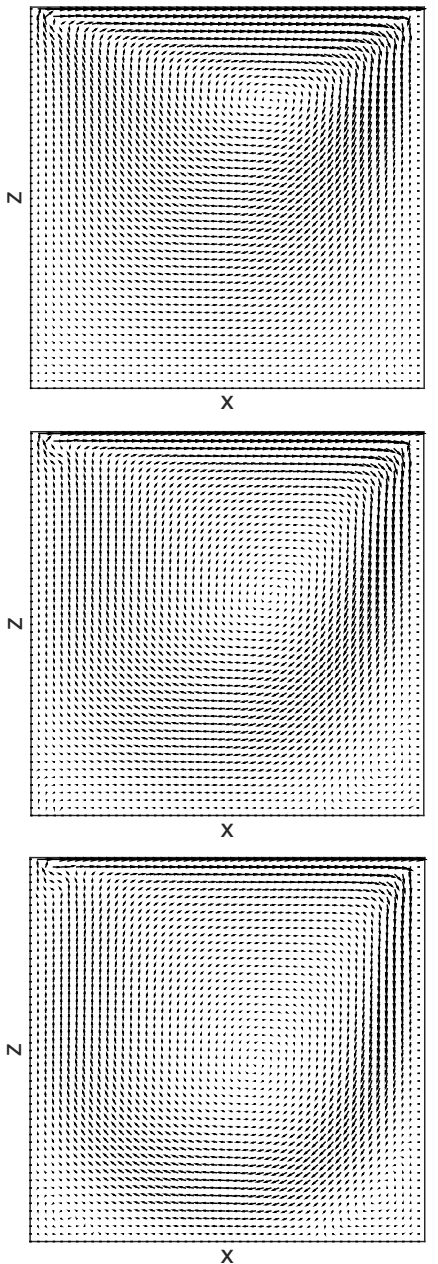
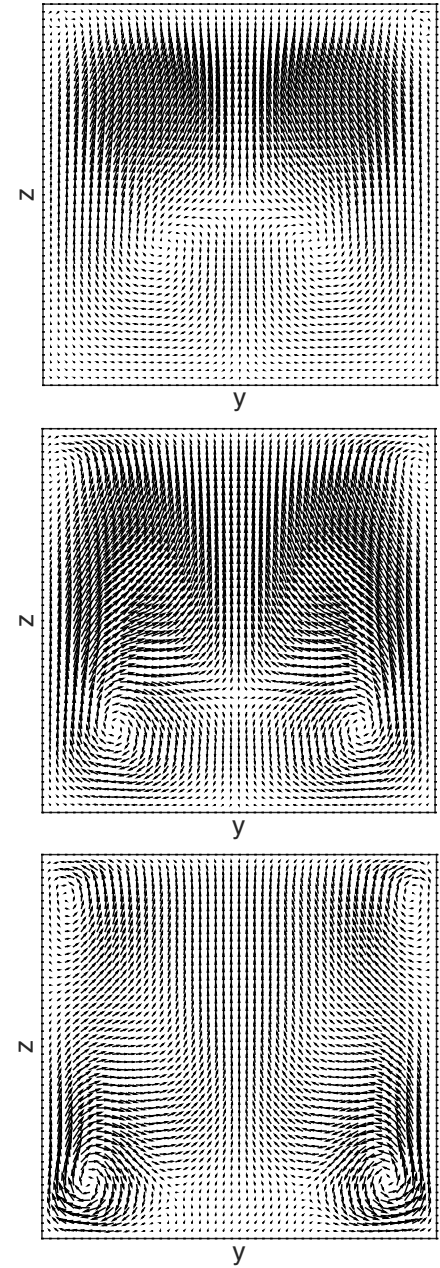

Figure 2: Shown above are the midsliceplane plots for the 3D driven cavity simulations at $\mathrm{Re}=100$, 400, and 1,000, using the Yosida-penalty method for the linear solves with $\gamma=1$ (and we note that identical plots are obtained for $\gamma=10,100$, and 1000). These plots are in excellent agreement with results reported in [31].

It is therefore worth comparing these methods, and we do so in this section. The key difference between the methods is for time-dependent problems $(\sigma>0)$; for steady problems, the methods give very similar solutions, but when $\sigma>0$, the $O\left(\sigma^{-2}\right)$ accuracy of the Yosida / Yosida-penalty method (and third order if pressure-correction step is used) provides a significant advantage over the iterated penalty method. After first presenting the classical iterated penalty method, we formulate new proofs based on pointwise divergence-free element spaces for (essentially) known convergence results, and finally compare them to results from the Yosida-penalty method using the 2D flow over a step problem used in the previous section. At the end of this section, we use the iterated penalty results to propose and discuss an iterated-penalty projection method for unsteady NS equations, and compare it qualitatively to the Yosida-penalty method.

Iterated penalty methods for solving Stokes and NSE problem have been used successfully for 

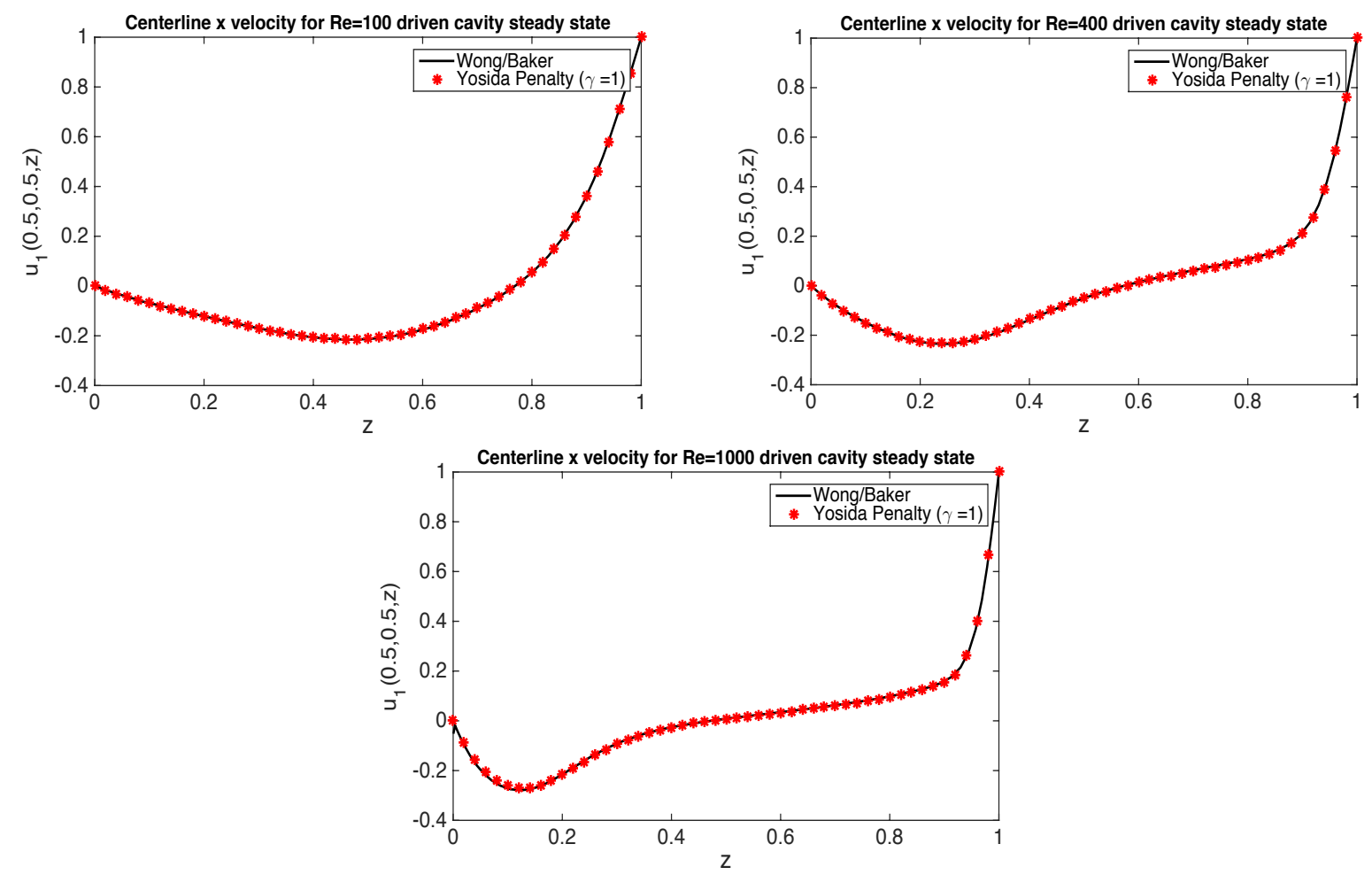

Figure 3: Shown above are the centerline $\mathrm{x}$-velocity plots for the $3 \mathrm{D}$ driven cavity simulations at $\operatorname{Re}=100,400$, and 1,000, using the penalty-Yosida method with $\gamma=1$ (and we note that identical plots are obtained for $\gamma=10,100$, and 1000). These plots are in excellent agreement with results reported in [31].

many years, see e.g. [32, 7, 14], and analysis of iterated penalty methods is generally done by transforming to a coupled system with artificial incompressibility / pressure regularization. Next, we analyze the methods directly, taking advantage of the divergence-free subspace $V_{h}$ and that the norm of its orthogonal complement in $X_{h}$ is the divergence norm.

\subsection{Iterated penalty method for the Stokes equations}

For simplicity, we consider first the Stokes equations, which are given by

$$
-\nu \Delta u+\nabla p=f, \quad \nabla \cdot u=0,
$$

and equipped with homogeneous boundary conditions for velocity (for simplicity), and zero-mean pressure. We will assume that the forcing $f \in H^{-1}(\Omega)$.

The standard coupled finite element approximation algorithm for the Stokes equations is as follows. Recall we assume that $\left(X_{h}, Q_{h}\right)$ is both LBB stable and $\nabla \cdot X_{h} \subset Q_{h}$ (e.g. Scott-Vogelius elements). 
Algorithm 4.1 (Pointwise divergence free solution for coupled Stokes problem).

Find $\left(u_{h}, p_{h}\right) \in\left(X_{h}, Q_{h}\right)$ satisfying

$$
\begin{aligned}
\nu\left(\nabla u_{h}, \nabla v_{h}\right)-\left(p_{h}, \nabla \cdot v_{h}\right) & =\left(f, v_{h}\right) \forall v_{h} \in X_{h}, \\
\left(\nabla \cdot u_{h}, q_{h}\right) & =0 \forall q_{h} \in Q_{h} .
\end{aligned}
$$

It is well known (see, e.g. [17]) that since LBB is assumed to hold for $\left(X_{h}, Q_{h}\right)$, Algorithm 4.1 is well posed and solutions are bounded as

$$
\left\|\nabla u_{h}\right\| \leq \nu^{-1}\|f\|_{-1}, \quad\left\|p_{h}\right\| \leq 2 \beta^{-1}\|f\|_{-1} .
$$

Consider now the classical iterated penalty algorithm, based on a formulation from [32] and in the same spirit as in $[19,14,7]$, but here we use exact integration in the penalty term.

Algorithm 4.2 (Iterated penalty method for Stokes).

Step 0: Find $w_{h}^{0} \in X_{h}$ satisfying

$$
\nu\left(\nabla w_{h}^{0}, \nabla v_{h}\right)+\gamma\left(\nabla \cdot w_{h}^{0}, \nabla \cdot v_{h}\right)=\left(f, v_{h}\right) \forall v_{h} \in X_{h} .
$$

Step $k=1,2, \ldots:$ Find $w_{h}^{k} \in X_{h}$ satisfying

$$
\nu\left(\nabla w_{h}^{k}, \nabla v_{h}\right)+\gamma\left(\nabla \cdot w_{h}^{k}, \nabla \cdot v_{h}\right)=\nu\left(\nabla w_{h}^{k-1}, \nabla v_{h}\right) \forall v_{h} \in X_{h} .
$$

Observe that Algorithm 4.2 produces, at step k,

$$
\begin{aligned}
\nu\left(\nabla w_{h}^{k}, \nabla v_{h}\right)+\gamma\left(\nabla \cdot w_{h}^{k}, \nabla \cdot v_{h}\right) & =\nu\left(\nabla w_{h}^{k-1}, \nabla v_{h}\right) \\
& =\nu\left(\nabla w_{h}^{k-2}, \nabla v_{h}\right)-\gamma\left(\nabla \cdot w_{h}^{k-1}, \nabla \cdot v_{h}\right) \\
& =\cdots \\
& =\nu\left(\nabla w_{h}^{0}, \nabla v_{h}\right)-\gamma\left(\nabla \cdot\left(\sum_{n=1}^{k-1} w_{h}^{n}\right), \nabla \cdot v_{h}\right),
\end{aligned}
$$

and thus

$$
\nu\left(\nabla w_{h}^{k}, \nabla v_{h}\right)+\gamma\left(\nabla \cdot\left(\sum_{n=0}^{k} w_{h}^{n}\right), \nabla \cdot v_{h}\right)=\left(f, v_{h}\right) \forall v_{h} \in X_{h} .
$$

Also, in step 0 , note that by choosing $v_{h}=w_{h}^{0}$, we get the bound

$$
\left\|\nabla w_{h}^{0}\right\| \leq \nu^{-1}\|f\|_{-1} .
$$

We prove now that the solution of the iterated penalty method converges linearly to the divergence free coupled method solution.

Theorem 4.1. Let $\left(u_{h}, p_{h}\right) \in\left(X_{h}, Q_{h}\right)$ be the coupled method solution of Algorithm 4.1, and $\left(w_{h}^{k}, \hat{p}_{h}^{k}\right) \in\left(X_{h}, Q_{h}\right), k=0,1,2, \ldots$, be the sequence of iterated penalty solutions of Algorithm 4.2, with $\hat{p}_{h}^{k}:=-\gamma \sum_{n=0}^{k}\left(\nabla \cdot w_{h}^{n}\right)$. Then

$$
\begin{gathered}
\left\|\nabla\left(u_{h}-w_{h}^{k}\right)\right\| \leq 2 \nu^{-1}\|f\|_{-1}\left(\frac{\sqrt{d} \nu C_{R}^{3}}{\gamma}\right)^{k}, \\
\left\|p_{h}-\hat{p}_{h}^{k}\right\| \leq 2 \beta^{-1}\|f\|_{-1}\left(\frac{\sqrt{d} \nu C_{R}^{3}}{\gamma}\right)^{k} .
\end{gathered}
$$


Proof. In this proof, we denote $e^{k}:=w_{h}^{k}-u_{h}$, and the pieces of $e^{k}$ resulting from the $H^{1}$ orthogonal decomposition as

$$
e^{k}=: e_{0}^{k}+e_{r}^{k}
$$

with $e_{0}^{k} \in V_{h}, e_{r}^{k} \in R_{h}$. The orthogonal decomposition of the test function $v_{h}$ will be done in an analogous manner: $v_{h}=v_{0}+v_{r}$.

We begin by subtracting (4.4) from (4.1), and noting that $\nabla \cdot u_{h}=0$ to get, for all $v_{h} \in X_{h}$,

$$
\nu\left(\nabla e^{0}, \nabla v_{h}\right)+\gamma\left(\nabla \cdot e^{0}, \nabla \cdot v_{h}\right)+\left(p_{h}, \nabla \cdot v_{h}\right)=0 .
$$

Note that choosing $v_{h}=e_{0}^{0} \in V_{h}$ implies that $\left\|\nabla e_{0}^{0}\right\|=0$.

Next, subtract $\nu\left(\nabla u_{h}, \nabla v_{h}\right)$ from both sides of (4.5) to get, for all $v_{h} \in X_{h}$,

$$
\nu\left(\nabla e^{k}, \nabla v_{h}\right)+\gamma\left(\nabla \cdot e^{k}, \nabla \cdot v_{h}\right)=\nu\left(\nabla e^{k-1}, \nabla v_{h}\right) .
$$

Restricting test functions to just those in the divergence free space $V_{h}$, we obtain

$$
\nu\left(\nabla e^{k}, \nabla v_{0}\right)=\nu\left(\nabla e^{k-1}, \nabla v_{0}\right) \forall v_{0} \in V_{h},
$$

and by orthogonality of $V_{h}$ and $R_{h}$,

$$
\left(\nabla\left(e_{0}^{k}-e_{0}^{k-1}\right), \nabla v_{0}\right)=0 \forall v_{0} \in V_{h} .
$$

Choosing $v_{0}=e_{0}^{k}-e_{0}^{k-1}$ now yields $\left\|\nabla\left(e_{0}^{k}-e_{0}^{k-1}\right)\right\|=0$, and in turn $e_{0}^{k}=e_{0}^{k-1}=\ldots=e_{0}^{0}$. Since $e_{0}^{0}=0$, we have proven that $e_{0}^{k}=0$ for all $k$, and it now remains to prove the convergence result for $e_{r}^{k}$.

We proceed by choosing $v_{h}=e_{r}^{k}$ in (4.8), which yields after using orthogonality of $V_{h}$ and $R_{h}$,

$$
\nu\left\|\nabla e_{r}^{k}\right\|^{2}+\gamma\left\|\nabla \cdot e_{r}^{k}\right\|^{2}=\nu\left(\nabla e_{r}^{k-1}, \nabla e_{r}^{k}\right) .
$$

Using Cauchy-Schwarz, Lemma 2.1, and Young's inequality on the right hand side produces

$$
\begin{aligned}
\nu\left\|\nabla e_{r}^{k}\right\|^{2}+\gamma\left\|\nabla \cdot e_{r}^{k}\right\|^{2} & \leq \nu\left\|\nabla e_{r}^{k-1}\right\|\left\|\nabla e_{r}^{k}\right\| \\
& \leq \nu C_{R}^{2}\left\|\nabla \cdot e_{r}^{k-1}\right\|\left\|\nabla \cdot e_{r}^{k}\right\| \\
& \leq \frac{\nu^{2} C_{R}^{4}}{2 \gamma}\left\|\nabla \cdot e_{r}^{k-1}\right\|^{2}+\frac{\gamma}{2}\left\|\nabla \cdot e_{r}^{k}\right\|^{2}
\end{aligned}
$$

and thus

$$
\left\|\nabla \cdot e_{r}^{k}\right\| \leq \frac{\nu C_{R}^{2}}{\gamma}\left\|\nabla \cdot e_{r}^{k-1}\right\| .
$$

Utilizing Lemma 2.1 once more, we obtain

$$
\left\|\nabla e_{r}^{k}\right\| \leq C_{R}\left\|\nabla \cdot e_{r}^{k}\right\| \leq \frac{\nu C_{R}^{3}}{\gamma}\left\|\nabla \cdot e_{r}^{k-1}\right\| \leq \frac{\sqrt{d} \nu C_{R}^{3}}{\gamma}\left\|\nabla e_{r}^{k-1}\right\|
$$

and combining the pieces of $e^{k}$ we get

$$
\left\|\nabla e^{k}\right\| \leq \frac{\sqrt{d} \nu C_{R}^{3}}{\gamma}\left\|\nabla e^{k-1}\right\| \leq \ldots \leq\left(\frac{\sqrt{d} \nu C_{R}^{3}}{\gamma}\right)^{k}\left\|\nabla e^{0}\right\| .
$$


Finally, applying the bounds (4.7) and (4.3) for $w_{h}^{0}$ and $u_{h}$ completes the proof for velocity.

For pressure, subtract (4.6) from (4.1) to get for all $v_{h} \in X_{h}$,

$$
\left(p_{h}-\hat{p}_{h}^{k}, \nabla \cdot v_{h}\right)=\nu\left(\nabla\left(w_{h}^{k}-u_{h}\right), \nabla v_{h}\right) .
$$

Applying Cauchy Schwarz on the right hand side, dividing both sides by $\left\|\nabla v_{h}\right\|$ and applying the inf-sup condition gives

$$
\left\|p_{h}-\hat{p}_{h}^{k}\right\| \leq \beta^{-1} \nu\left\|\nabla\left(w_{h}^{k}-u_{h}\right)\right\| .
$$

Now using the velocity estimate completes the proof.

\subsection{Extension to Navier-Stokes equations}

We consider next iterated penalty methods for the (steady or time dependent) NS equations. We use the same continuous and coercive bilinear forms used throughout the paper:

$$
a(u, v):=\sigma(u, v)+\nu(\nabla u, \nabla v)+b^{*}(U, u, v)=\widetilde{a}(u, v)+b^{*}(U, u, v),
$$

where $U$ is known, $\sigma=0$ for steady problems, and $\sigma>0$ for time dependent problems. The finite element problem of interest remains Algorithm 3.1, and the associated iterated penalty method reads as follows.

Algorithm 4.3 (Iterated penalty method for NS).

Step 0: Find $w_{h}^{0} \in X_{h}$ satisfying

$$
a\left(w_{h}^{0}, v_{h}\right)+\gamma\left(\nabla \cdot w_{h}^{0}, \nabla \cdot v_{h}\right)=\left(f, v_{h}\right) \forall v_{h} \in X_{h} .
$$

Step $k=1,2, \ldots:$ Find $w_{h}^{k} \in X_{h}$ satisfying

$$
a\left(w_{h}^{k}, v_{h}\right)+\gamma\left(\nabla \cdot w_{h}^{k}, \nabla \cdot v_{h}\right)=a\left(w_{h}^{k-1}, v_{h}\right) \forall v_{h} \in X_{h} .
$$

We can now state a convergence result for the NS iterated penalty method.

Theorem 4.2. Let $\left(u_{h}, p_{h}\right) \in\left(X_{h}, Q_{h}\right)$ be the coupled method solution of Algorithm 3.1, and $\left(w_{h}^{k}, \hat{p}_{h}^{k}\right) \in\left(X_{h}, Q_{h}\right), k=0,1,2, \ldots$, be the sequence of iterated penalty solutions of Algorithm 4.3, with $\hat{p}_{h}^{k}:=-\gamma \sum_{n=0}^{k}\left(\nabla \cdot w_{h}^{n}\right)$. Then for $K=C_{\tilde{a}}^{2}\left(1+C_{s} C_{U} \nu^{-1}\left(1+C_{s} C_{U} \nu^{-1}\right)\right)$,

$$
\begin{gathered}
\left\|u_{h}-w_{h}^{k}\right\|_{\tilde{a}} \leq \frac{C_{P}}{\gamma}\left(\frac{\left(K+C_{\tilde{a}}^{2}\right) C_{\tilde{a}}\left(1+C_{s} C_{U} \nu^{-1}\right)}{\gamma}\right)^{k}, \\
\left\|p_{h}-\hat{p}_{h}^{k}\right\| \leq \frac{\beta^{-1} C_{P}}{\gamma}\left(\frac{\left(K+C_{\tilde{a}}^{2}\right) C_{\tilde{a}}\left(1+C_{s} C_{U} \nu^{-1}\right)}{\gamma}\right)^{k} .
\end{gathered}
$$

Thus for $\gamma$ sufficiently large, the errors converge linearly with $k$.

Proof. This proof follows by combining ideas from the Stokes iterated penalty proof above, and from the Yosida-penalty analysis of the previous section. The main difference from the Stokes proof is that the orthogonal decomposition is done with the $\tilde{a}$ inner product, which leads to some $b^{*}$ terms arising in the analysis. These terms are handled analogous to the Yosida-penalty method proof. 


\subsection{Numerical experiments}

We now compare the Yosida-penalty method to the iterated penalty method, using the same test problem as above for 2D flow over a step at $R e=20$, with varying $\gamma$, first with $\sigma=0$ and then with $\sigma=1$. Since the Yosida-penalty method used 2 iterated-penalty-type steps, we compare its solution with that of the iterated penalty solution after two steps. Both of these methods are proven herein to be $O\left(\gamma^{-2}\right)$ as $\gamma \rightarrow \infty$. Whether this is a 'fair' comparison depends strongly on whether the cost of the SPD Schur complement solve is negligible; for larger $\gamma$ and for time dependent problems, this can be a reasonable assumption since the Schur complement is not time dependent (and so preconditioners only need built once) and the iteration count for the Schur complement solve is small when $\gamma \geq 10$ (usually less than 4 or 5 iterations of CG). To quantify accuracy, we compare solutions to the coupled method solution with the same $\sigma$.

The results for $\sigma=0$ are shown in table 5, and indicate almost identical accuracy between the Yosida-penalty method and 2 steps of the iterated penalty method, for each $\gamma$. The results for $\sigma=1$ are shown in table 6 , and are much different: here, the Yosida-penalty method is one to two orders of magnitude more accurate than the 2-step-iterated penalty method, for both the velocity and pressure. Since the Yosida-penalty method is still a Yosida method, it enjoys the theory of [28], where it is proven that the method is also $O\left(\sigma^{-2}\right)$. The iterated penalty method, on the other hand, does not improve as $\sigma$ increases; in fact, it gets worse, likely due to the constant $C_{\tilde{a}}$ increasing as $\sigma$ increases.

\begin{tabular}{|c||c|c||c|c||c|c|}
\hline$\gamma$ & $\left\|\nabla\left(u_{Y}^{\gamma}-u_{S V}\right)\right\|$ & $\left\|\nabla\left(u_{I t, 2}^{\gamma}-u_{S V}\right)\right\|$ & $\left\|\nabla \cdot u_{Y}^{\gamma}\right\|$ & $\left\|\nabla \cdot u_{I t, 2}^{\gamma}\right\|$ & $\left\|\hat{p}_{Y}^{\gamma}-p_{S V}\right\|$ & $\left\|\hat{p}_{I t, 2}^{\gamma}-p_{S V}\right\|$ \\
\hline 1 & $8.11 \mathrm{e}-0$ & $9.15 \mathrm{e}-1$ & $1.83 \mathrm{e}-1$ & $2.09 \mathrm{e}-1$ & $6.39 \mathrm{e}-1$ & $7.53 \mathrm{e}-1$ \\
\hline 10 & $1.44 \mathrm{e}-1$ & $1.71 \mathrm{e}-1$ & $2.47 \mathrm{e}-2$ & $2.71 \mathrm{e}-2$ & $1.14 \mathrm{e}-1$ & $1.30 \mathrm{e}-1$ \\
\hline 100 & $1.83 \mathrm{e}-3$ & $2.17 \mathrm{e}-3$ & $3.85 \mathrm{e}-4$ & $4.17 \mathrm{e}-4$ & $1.85 \mathrm{e}-3$ & $1.93 \mathrm{e}-3$ \\
\hline 1,000 & $1.85 \mathrm{e}-5$ & $2.17 \mathrm{e}-5$ & $3.97 \mathrm{e}-6$ & $4.28 \mathrm{e}-6$ & $1.93 \mathrm{e}-5$ & $1.99 \mathrm{e}-5$ \\
\hline 10,000 & $1.86 \mathrm{e}-7$ & $2.18 \mathrm{e}-7$ & $3.98 \mathrm{e}-8$ & $4.29 \mathrm{e}-8$ & $1.97 \mathrm{e}-7$ & $2.00 \mathrm{e}-7$ \\
\hline
\end{tabular}

Table 5: Differences between the SV solution and Yosida-penalty solutions, and 2-step-iteratedpenalty solutions, with varying $\gamma$ for the flow over a step benchmark problem with $\sigma=0$.

\begin{tabular}{|c||c|c||c|c||c|c|}
\hline$\gamma$ & $\left\|\nabla\left(u_{Y}^{\gamma}-u_{S V}\right)\right\|$ & $\left\|\nabla\left(u_{I t, 2}^{\gamma}-u_{S V}\right)\right\|$ & $\left\|\nabla \cdot u_{Y}^{\gamma}\right\|$ & $\left\|\nabla \cdot u_{I t, 2}^{\gamma}\right\|$ & $\left\|\hat{p}_{Y}^{\gamma}-p_{S V}\right\|$ & $\left\|\hat{p}_{I t, 2}^{\gamma}-p_{S V}\right\|$ \\
\hline 1 & $7.27 \mathrm{e}-1$ & $8.51 \mathrm{e}-0$ & $5.80 \mathrm{e}-1$ & $1.79 \mathrm{e}+0$ & $1.24 \mathrm{e}-0$ & $1.61 \mathrm{e}+2$ \\
\hline 10 & $2.41 \mathrm{e}-1$ & $7.23 \mathrm{e}-0$ & $1.01 \mathrm{e}-1$ & $9.55 \mathrm{e}-1$ & $1.58 \mathrm{e}-0$ & $1.44 \mathrm{e}+2$ \\
\hline 100 & $6.41 \mathrm{e}-2$ & $3.15 \mathrm{e}-0$ & $1.54 \mathrm{e}-2$ & $3.77 \mathrm{e}-1$ & $5.99 \mathrm{e}-1$ & $6.43 \mathrm{e}+1$ \\
\hline 1000 & $2.19 \mathrm{e}-3$ & $1.69 \mathrm{e}-1$ & $5.04 \mathrm{e}-4$ & $2.01 \mathrm{e}-2$ & $2.09 \mathrm{e}-2$ & $3.44 \mathrm{e}-0$ \\
\hline 10,000 & $2.64 \mathrm{e}-5$ & $2.23 \mathrm{e}-3$ & $6.07 \mathrm{e}-6$ & $2.67 \mathrm{e}-4$ & $2.53 \mathrm{e}-4$ & $4.56 \mathrm{e}-2$ \\
\hline
\end{tabular}

Table 6: Differences between the SV solution and Yosida-penalty solutions, and 2-step-iteratedpenalty solutions, with varying $\gamma$ for the flow over a step benchmark problem with $\sigma=1$. 


\subsection{Iterated penalty-projection method for transient Navier-Stokes}

The ideas and results of this section can be extended, although with more technical details, to create an iterated-penalty projection method that has similar cost as Yosida-penalty, and also converges to the coupled method solution with rate $O\left(\gamma^{-2}\right)$. Penalty-projection methods can be quite effective when larger grad-div stabilization parameters are used [20,16], and thus iterated-penalty projection methods could offer similar accuracy, but trade-off smaller parameters for additional solves. To define the projection methods, we first define an additional space for $H^{1}$ velocity functions, where only the normal component vanishes on the boundary,

$$
Y:=\left\{v \in H^{1}(\Omega),\left.v \cdot n\right|_{\partial \Omega}=0\right\} .
$$

Let $Y_{h} \subset Y$ be a finite element space, created exactly the same as $X_{h}$, expect for the boundary condition.

Consider first the standard BDF2 penalty-projection method.

Algorithm 4.4 (BDF2 penalty-projection).

Step 1: Find $u_{h}^{n+1} \in X_{h}$ satisfying, for all $\chi_{h} \in X_{h}$,

$$
\begin{aligned}
\frac{3}{2 \Delta t}\left(u_{h}^{n+1}, \chi_{h}\right) & +b^{*}\left(2 u_{h}^{n}-u_{h}^{n-1}, u_{h}^{n+1}, \chi_{h}\right)+\nu\left(\nabla u_{h}^{n+1}, \nabla \chi_{h}\right)+\gamma\left(\nabla \cdot u_{h}^{n+1}, \nabla \cdot \chi_{h}\right) \\
& =\left(f\left(t^{n+1}\right)+\frac{2}{\Delta t} \widetilde{u}_{h}^{n}-\frac{1}{2 \Delta t} \widetilde{u}_{h}^{n-1}, \chi_{h}\right)+\left(p_{h}^{n}, \nabla \cdot \chi_{h}\right) .
\end{aligned}
$$

Step 2: Find $\left(\widetilde{u}_{h}^{n+1}, p_{h}^{n+1}\right) \in\left(Y_{h}, Q_{h}\right)$ satisfying, for all $\left(w_{h}, q_{h}\right) \in\left(Y_{h}, Q_{h}\right)$,

$$
\begin{aligned}
\frac{3}{2 \Delta t}\left(\widetilde{u}_{h}^{n+1}-u_{h}^{n+1}, w_{h}\right)-\left(p_{h}^{n+1}-p_{h}^{n}, \nabla \cdot w_{h}\right) & =0 \\
\left(\nabla \cdot \widetilde{u}_{h}^{n+1}, q_{h}\right) & =0 .
\end{aligned}
$$

This method converges to the Algorithm 3.1 solution with rate $O\left(\gamma^{-1}\right)$ (this can be shown following techniques as in [20] for a similar scheme). However, this method is less costly than Yosida-penalty. Step 1 involves one solve of the form $A u=f$, and Step 2 can be solved with a SPD Schur complement solve, using $-B^{T} M B$ where $M$ is the velocity mass matrix. We note that if the Yosida-penalty method was used with an analogous (coupled) linearized BDF2 timestepping scheme, then the $A$ matrix would be the same in Yosida-penalty as it is for the Step 1 solve, but we would need to solve two linear systems that use the $A$ matrix, and one SPD Schur complement solve. Hence, a more appropriate comparison of methods that are of (closer to) equal cost would be comparing Yosida-penalty to an iterated penalty-projection method, where (4.11) above is split into two steps of the penalty iteration:

Algorithm 4.5 (BDF2 iterated penalty-projection). Step 1: Find $\hat{u}_{h}^{n+1} \in X_{h}$ satisfying, for all $\chi_{h} \in X_{h}$,

$$
\begin{aligned}
\frac{3}{2 \Delta t}\left(\hat{u}_{h}^{n+1}, \chi_{h}\right) & +b^{*}\left(2 u_{h}^{n}-u_{h}^{n-1}, \hat{u}_{h}^{n+1}, \chi_{h}\right)+\nu\left(\nabla \hat{u}_{h}^{n+1}, \nabla \chi_{h}\right)+\gamma\left(\nabla \cdot \hat{u}_{h}^{n+1}, \nabla \cdot \chi_{h}\right) \\
& =\left(f\left(t^{n+1}\right)+\frac{2}{\Delta t} \widetilde{u}_{h}^{n}-\frac{1}{2 \Delta t} \widetilde{u}_{h}^{n-1}, \chi_{h}\right)+\left(p_{h}^{n}, \nabla \cdot \chi_{h}\right) .
\end{aligned}
$$


Step 2: Find $u_{h}^{n+1} \in X_{h}$ satisfying, for all $\chi_{h} \in X_{h}$,

$$
\begin{aligned}
\frac{3}{2 \Delta t}\left(u_{h}^{n+1}, \chi_{h}\right) & +b^{*}\left(2 u_{h}^{n}-u_{h}^{n-1}, u_{h}^{n+1}, \chi_{h}\right)+\nu\left(\nabla u_{h}^{n+1}, \nabla \chi_{h}\right)+\gamma\left(\nabla \cdot u_{h}^{n+1}, \nabla \cdot \chi_{h}\right) \\
& =\frac{3}{2 \Delta t}\left(\hat{u}_{h}^{n+1}, \chi_{h}\right)+b^{*}\left(2 u_{h}^{n}-u_{h}^{n-1}, \hat{u}_{h}^{n+1}, \chi_{h}\right)+\nu\left(\nabla \hat{u}_{h}^{n+1}, \nabla \chi_{h}\right) .
\end{aligned}
$$

Step 3: Find $\left(\widetilde{u}_{h}^{n+1}, p_{h}^{n+1}\right) \in\left(Y_{h}, Q_{h}\right)$ satisfying, for all $\left(w_{h}, q_{h}\right) \in\left(Y_{h}, Q_{h}\right)$,

$$
\begin{aligned}
\frac{3}{2 \Delta t}\left(\widetilde{u}_{h}^{n+1}-u_{h}^{n+1}, w_{h}\right)-\left(p_{h}^{n+1}-p_{h}^{n}, \nabla \cdot w_{h}\right) & =0, \\
\left(\nabla \cdot \widetilde{u}_{h}^{n+1}, q_{h}\right) & =0 .
\end{aligned}
$$

Combining the iterated penalty analysis of this section with the analysis of [20] will (we expect) lead to $O\left(\gamma^{-2}\right)$ convergence of the solution of Algorithm 4.5 to the solution of Algorithm 3.1. Of course, the accuracy of these methods depends on constants other than $\gamma$, most importantly the timestep $\Delta t$, and so for a particular finite $\gamma$, it is not clear whether a Yosida-penalty method would be better than Algorithm 4.5; likely, it is problem dependent. Moreover, the way in which these different methods 'split' the systems creates error in different ways. While (pressure-correction) projection methods approximation error arises from the pressure term, the Yosida approximation error comes from (at least) the nonlinearity, since we take $N=0$ to create $\widetilde{A}$ from $A$ (but there are other ways to approximate). Formally, the pressure and nonlinearity are related via $\Delta p=$ $-\nabla \cdot(u \cdot \nabla u)$ ), which can be seen by taking divergence of the NS momentum equation. Still, knowing which terms might be dominant in a simulation can help to determine which splitting method could be a better choice. The authors plan to explore this idea and comparisons in future work.

\section{Conclusions}

We have proven that in the setting where divergence free elements are LBB stable, finite element solutions of incompressible flow problems found using the Yosida-penalty method converge to the divergence-free solution (i.e. the solution found by solving the linear system exactly), with rate $O\left(\gamma^{-2}\right)$ as $\gamma \rightarrow \infty$. The proof was based on i) using grad-div stabilization (with penalty parameter $\gamma$ ) together with divergence-free elements, ii) transforming the inexact block LU factorization produced by the Yosida method into a series of finite element problems, and iii) using finite element techniques based on pointwise divergence-free subspaces and their orthogonal complements to analyze the series of finite element problems.

Several numerical tests were given to confirm the theory, and which also suggest that $\gamma=10$ is sufficient in the Yosida-penalty method to achieve accuracy very close to that of the divergencefree coupled method solution of Algorithm 3.1. Further, with $\gamma \geq 10$, the number of outer CG iterations needed to resolve linear systems with the SPD approximated Schur complement $\widetilde{S}$ to a tolerance of $1 e-10$ was always less than 5 , and decreased for larger $\gamma$. We also observed that with smaller $\gamma$, accuracy can be increased in time dependent problems by using the pressure-corrected Yosida-penalty method, although this alteration of the Yosida-penalty method does not increase the convergence rate with respect to $\gamma$. 


\section{Acknowledgement}

The authors wish to thank Prof. Alessandro Veneziani for several insightful discussions about Yosida methods.

\section{References}

[1] D. Arnold and J. Qin. Quadratic velocity/linear pressure Stokes elements. In R. Vichnevetsky, D. Knight, and G. Richter, editors, Advances in Computer Methods for Partial Differential Equations VII, pages 28-34. IMACS, 1992.

[2] M. Benzi, G. Golub, and J. Liesen. Numerical solution of saddle point problems. Acta Numerica, 14:1-137, 2005.

[3] M. Benzi and M. Olshanskii. An augmented Lagrangian-based approach to the Oseen problem. SIAM J. Sci. Comput., 28:2095-2113, 2006.

[4] D. Brown, R. Cortez, and M. Minion. Accurate projection methods for the incompressible Navier-Stokes equations. Journal of Computational Physics, 168:464-499, 2001.

[5] C. Canuto, M.Y. Hussaini, A. Quarteroni, and T.A.Zang. Spectral Methods: Evolution to Complex Geometries and Applications to Fluid Dynamics. Springer, Berlin, 2007.

[6] A. J. Chorin. Numerical solution for the Navier-Stokes equations. Math. Comp., 22:745-762, 1968.

[7] R. Codina. An iterative penalty method for the finite element solution of the stationary Navier-Stokes equations. Computer Methods in Applied Mechanics and Engineering, 110:237262, 1993.

[8] H. Elman, D. Silvester, and A. Wathen. Finite Elements and Fast Iterative Solvers with applications in incompressible fluid dynamics. Numerical Mathematics and Scientific Computation. Oxford University Press, Oxford, 2005.

[9] R. Falk and M. Neilan. Stokes complexes and the construction of stable finite elements with pointwise mass conservation. SIAM J. Numer. Anal., 51(2):1308-1326, 2013.

[10] L. Franca and T. Hughes. Two classes of mixed finite element methods. Computer Methods in Applied Mechanics and Engineering, 69(1):89-129, 1988.

[11] P. Gervasio, F. Saleri, and A. Veneziani. Algebraic fractional-step schemes with spectral methods for the incompressible Navier-Stokes equations. Journal of Computational Physics, 214:347-365, 2006.

[12] V. Girault and P.-A. Raviart. Finite element methods for Navier-Stokes equations: Theory and algorithms. Springer-Verlag, 1986.

[13] J. Guermond, P. Minev, and J. Shen. An overview of projection methods for incompressible flows. Computer Methods in Applied Mechanics and Engineering, 195:6011-6045, 2006. 
[14] M. Gunzburger. Iterative penalty methods for the Stokes and Navier-Stokes equations. Proceedings from Finite Element Analysis in Fluids conference, University of Alabama, Huntsville, pages 1040-1045, 1989.

[15] J. Guzmán and M. Neilan. Conforming and divergence-free Stokes elements on general triangulations. Math. Comp., 83:15-36, 2014.

[16] M. Jobelin, C. Lapuerta, J.-C. Latche, Ph. Angot, and B. Piar. A finite element penaltyprojection method for incompressible flows. Journal of Computational Physics, 217:502-518, 2006.

[17] W. Layton. An Introduction to the Numerical Analysis of Viscous Incompressible Flows. SIAM, Philadelphia, 2008.

[18] Y. Lee, J. Wu, and J. Chen. Robust multi grid method for the planar linear elasticity problems. Numer. Math., 113:473-496, 2009.

[19] S. Lin, Y. Chin, and T. Wu. A modified penalty method for Stokes equations and applications to Navier-Stokes equations. SIAM Journal on Scientific Computing, 16:1-19, 1995.

[20] A. Linke, M. Neilan, L. Rebholz, and N. Wilson. Improving efficiency of coupled schemes for Navier-Stokes equations by a connection to grad-div stabilized projection methods. Submitted, 2014.

[21] M. A. Olshanskii and A. Reusken. Grad-Div stabilization for the Stokes equations. Math. Comp., 73:1699-1718, 2004.

[22] M.A. Olshanskii and E.E. Tyrtyshnikov. Iterative Methods for Linear Systems: Theory and Applications. SIAM, Philadelphia, 2014.

[23] J. Perot. An analysis of the fractional step method. Journal of Computational Physics, 108(1):51-58, 1993.

[24] A. Prohl. Projection and quasi-compressibility methods for solving the incompressible NavierStokes equations. Teubner-Verlag, Stuttgart, 1997.

[25] A. Prohl. On pressure approximation via projection methods for nonstationary incompressible Navier-Stokes equations. SIAM Journal on Numerical Analysis, 47(1):158-180, 2008.

[26] A. Quarteroni, F. Saleri, and A. Veneziani. Analysis of the Yosida method for incompressible Navier-Stokes equations. J. Math. Pures. Appl., 78:473-503, 1999.

[27] A. Quarteroni, F. Saleri, and A. Veneziani. Factorization methods for the numerical approximation of Navier-Stokes equations. Computer Methods in Applied Mechanics and Engineering, 188:505-526, 2000.

[28] F. Saleri and A. Veneziani. Pressure correction algebraic splitting methods for the incompressible Navier-Stokes equations. SIAM Journal on Numerical Analysis, 43:174-194, 2006.

[29] J. Schöberl. Robust multigrid methods for a parameter dependent problem in primal variables. Numerische Mathematik, 84:97-119, 1999. 
[30] R. Temam. Sur l'approximation de la solution des equations de Navier-Stokes par la methode des pas fractionnaires (II). Arch. Rational Mech. Anal., 33:377-385, 1969.

[31] K.L. Wong and A.J. Baker. A 3d incompressible Navier-Stokes velocity-vorticity weak form finite element algorithm. International Journal for Numerical Methods in Fluids, 38:99-123, 2002 .

[32] C. Xiao-liang and A. Shaikh. Analysis of the iterative penalty method for the stokes equations. Applied Mathematics Letters, 19:1024-1028, 2006.

[33] S. Zhang. A new family of stable mixed finite elements for the 3d Stokes equations. Math. Comp., 74(250):543-554, 2005. 